\title{
ESTIMATION OF INTERLAMINAR STRESSES IN FIBRE REINFORCED COMPOSITE CYLINDRICAL SHELLS
}

\author{
T. Kant† and M. P. MENON \\ Department of Civil Engineering, Indian Institute of Technology, Powai, Bombay 400 076, India
}

(Received 19 November 1989)

\begin{abstract}
A C^{0}$ finite element formulation for flexure-membrane coupling behavior of symmetric and asymmetric laminated cylindrical shells based on a higher-order displacement model is presented. This theory incorporates a realistic nonlinear variation of displacements through the shell thickness, and eliminates the use of shear correction coefficient/s. The discrete element chosen is a nine-noded quadrilateral with nine degrees of freedom per node.

The solutions are obtained through two formulations: (1) the geometrically thin shell formulation, based on the assumption that the ratio of thickness to radius of the shell is very much less than unity, and (2) the geometrically thick shell formulation, in which $(h / R)^{2} \ll 1$. In these formulations, the in-plane stresses are obtained via constitutive relations. Reliable estimates of interlaminar stresses from equilibrium equations are obtained. A finite difference scheme maintaining the continuity of interlaminar stresses across the shell thickness is developed and used. The results obtained are compared with available elasticity, closed-form and other finite element solutions.
\end{abstract}

\section{INTRODUCTION}

Multilayered fibre reinforced composite shells of revolution have found increasing applications in aerospace industries, as they offer high strength-to-weight and stiffness-to-weight ratios. The increased use of these materials has been the moving force for much research activity in recent years. One subject of such studies, failure due to delamination, is of considerable importance. This involves separation of individual laminae, especially at the free edges, caused by low strength along the ply interfaces and high local interlaminar stresses. This results in structural and functional failure as a result of the destruction of the load-transferring mechanism. The initiation and growth of this failure is related to the interlaminar stresses acting through the shell thickness. Further, the in-plane lamina stresses are necessary to ensure that the strength requirements of the lamina are fulfilled. Thus a theory which can predict all these stresses accurately becomes necessary for understanding the failure mechanism of fiber reinforced composite shells.

The finite element method is most convenient for finding a solution for general laminated composite shells which have complex geometries, arbitrary loadings and boundary conditions.

In a shell theory, a three-dimensional system is reduced to a two-dimensional one by incorporating simplifying assumptions. In the classical Love's thin shell theory, based on Kirchhoff's hypothesis [1], the

† To whom all correspondence should be addressed. laminae are assumed to be in a state of plane stress and the effects of transverse shear and normal strains in the thickness direction are not considered. Klosner and Levinc [2] have investigated different shell theories and have compared them with the three-dimensional elasticity solution for an isotropic infinite circular cylindrical shell subjected to periodically spaced band loads. Ambartsumyan [3] appears to have been the first to observe the coupling between stretching and bending inherent in laminated anisotropic shells.

It has been observed in the literature that many of the theories for analysing the laminated shell have been based on classical laminate theory, incorporating the Love-Kirchhoff hypothesis. These theories have been reviewed in [4-6]. Dong and Taylor [7] have extended Donnell's shallow shell theory and formulated a theory for a thin laminated anisotropic shell. A first approximation theory by Widera and Chung [8] has been derived for asymmetric deformations of a nonhomogeneous anisotropic cylindrical shell, using the asymptotic integration of the elasticity equations. Reuter [9] has used Donnell's shallow shell theory, in obtaining closed-form solutions for unbalanced symmetric and balanced asymmetric angle-ply cylindrical shells. Balaraman et al. [10] have obtained closed-form solutions for an asymmetrically laminated cylindrical shell of orthotropic as well as anisotropic layers under uniform pressure. A closed-form solution for an arbitrary laminated anisotropic cylindrical shell based on the classical laminate theory has been given by Chaudhuri [11]. Most of the above analyses are based on the assumption that the transverse shear 
strains are negligible, and thus hold good for thin shells.

Dong and Tso [12] were perhaps the first to present a first-order shear deformation theory, which included the effect of transverse shear deformation through the shell thickness; they then constructed a laminated orthotropic shell theory. This theory can be assumed as an extension of Love's first approximation theory for homogeneous isotropic shell. However, only a cylindrical shell in which the orthotropic axes of each layer coincide with the reference axes of the shell can be analysed with this theory.

A bending theory known as the moderately thick plate/shell theory or constant shear angle theory and based on the hypothesis of constant shear angle through its thickness, is an extension of Mindlin's shear deformation theory [13] for a homogeneous isotropic plate. Series solutions of these equations for cross-ply cylindrical and doubly curved shells have been presented by Reddy [14]. However, Reddy concluded that these serics solutions do not give good results for an antisymmetric angle-ply shallow shell.

A layerwise constant shear angle assumption has been used to solve plate/shell problems by Seide and Chaudhuri[15], with quadratic triangular element formulation. Kamal [16] has obtained exact solutions for an arbitrarily laminated, anisotropic, axisymmetrical cylindrical shell of finite length and subjected to internal pressure, based on the constant transverse shear deformation theory. In another refined theory, Whitney and Sun [17] derived a set of governing equations and boundary conditions which include both transverse shear deformation and transverse normal strain in a laminated anisotropic cylindrical shell.

Second-order shear deformation effects have been included by Kant [18], who has developed governing equilibrium equations for a thick shell which are applicable to an orthotropic material with planes of symmetry which coincide with the orthogonal reference plane. Bhimaraddi [19] has presented a refined theory for a cylindrical shell, assuming a displacement function which results in parabolic variation of transverse shear strains and also satisfies shear-free surface boundary conditions on the bounding surfaces. Bhimaraddi achieved this by introducing a function $\xi(z)$ in the displacement expression, whose first derivative vanishes at the extreme fibers. In another paper, Bhimaraddi and Stevens [20] have given other possible forms of $\xi(x)$-functions. Murty and Reddy [21] have proposed a higher-order theory for the analysis of a composite cylindrical shell. The formulation allows for arbitrary variation of in-plane displacement, and the formulation results in $C^{1}$ continuity.

In the theories which use a constant shear angle or a piecewise constant shear angle through the thickness, shear correction coefficient/s whose accurate prediction for anisotropic laminated shell is cumbersome and problem dependent is/are used. The effect of true cross-sectional warping, which must be considered in the case of sandwich shells, is not taken into account in these theories. Thus a refined theory which considers a realistic parabolic variation of transverse shear stresses through the thickness is essential. A $C^{0}$ finite element formulation based on a higher-order displacement model, incorporating the effect of transverse shear deformation and suitable for the analysis of a thin as well as a moderately thick laminated anisotropic cylindrical shell, is developed here. In this paper, emphasis is placed on the evaluation of interlaminar stresses by finite difference methods, satisfying the equilibrium equations and thus maintaining the continuity of stresses at the interface of layers through the thickness.

\section{THEORY AND FORMULATION}

To approximate the three-dimensional elasticity problem as a two-dimensional problem, the displacement components $U_{i}(\theta, x, z), i=1,3$, at any point in the shell are expanded in a Taylor series in terms of the thickness coordinate. The elasticity solution indicates that the transverse shear stress varies parabolically through the shell thickness. This requires the use of a displacement field in which the in-plane displacements are expanded as a cubic function of the thickness coordinate. The displacement field which satisfies the above criteria may be assumed in the form

$$
\begin{aligned}
& U_{i}=u_{i}+z \theta_{i}+z^{2} u_{i}^{*}+z^{3} \theta_{i}^{*}, \quad i=1,2 \\
& U_{3}=u_{3},
\end{aligned}
$$

where the displacements $U_{i}(i=1,3)$ are at any point at a distance $z$ from the reference surface. The mid-surface of the shell is treated as this reference surface, and

$$
\delta^{s}=\left(u_{1}, u_{2}, u_{3}, \theta_{1}, \theta_{2}, u_{1}^{*}, u_{2}^{*}, \theta_{1}^{*}, \theta_{2}^{*}\right)^{t}
$$

are the reference surface displacements and rotations. Equation (1a) contains minimum numbers of terms to include the effect of transverse shear deformation with warping of the transverse normal crosssection.

By substituting these relations into the straindisplacement equations $[1,18]$ and specializing these for a cylindrical shell, the following relations are obtained.

Geometrically thin shell:

$$
\begin{aligned}
\epsilon_{i}^{z} & =\left(\epsilon_{i}+z x_{i}+z^{2} \epsilon_{i}^{*}+z^{3} \varkappa_{i}^{*}\right),(i=1,2) \\
\gamma_{12}^{z} & =\left(\epsilon_{12}+z x_{12}+z^{2} \epsilon_{12}^{*}+z^{3} x_{12}^{*}\right) \\
\gamma_{i 3}^{z} & =\left(\phi_{i}+z \psi_{i}+z^{2} \phi_{i}^{*}\right),(i=1,2),
\end{aligned}
$$


where

$$
\begin{aligned}
{\left[\epsilon_{1}, \epsilon_{1}^{*}, \chi_{1}, \chi_{1}^{*}\right]=} & {\left[\frac{\partial u_{1}}{R \partial \theta}+\frac{u_{3}}{R}, \frac{\partial u_{1}^{*}}{R \partial \theta}, \frac{\partial \theta_{1}}{R \partial \theta}, \frac{\partial \theta_{1}^{*}}{R \partial \theta}\right] } \\
{\left[\epsilon_{2}, \epsilon_{2}^{*}, \chi_{2}, \chi_{2}^{*}\right]=} & {\left[\frac{\partial u_{2}}{\partial x}, \frac{\partial u_{2}^{*}}{\partial x}, \frac{\partial \theta_{2}}{\partial x}, \frac{\partial \theta_{2}^{*}}{\partial x}\right] } \\
{\left[\epsilon_{12}, \epsilon_{12}^{*}, \chi_{12}, \chi_{12}^{*}\right]=} & {\left[\frac{\partial u_{2}}{R \partial \theta}+\frac{\partial u_{1}}{\partial x}, \frac{\partial u_{2}^{*}}{R \partial \theta}\right.} \\
& \left.+\frac{\partial u_{1}^{*}}{\partial x}, \frac{\partial \theta_{2}}{R \partial \theta}+\frac{\partial \theta_{1}}{\partial x}, \frac{\partial \theta_{2}^{*}}{R \partial \theta}+\frac{\partial \theta_{1}^{*}}{\partial x}\right] \\
{\left[\phi_{1}, \psi_{1}, \phi_{1}^{*}\right]=} & {\left[\frac{\partial u_{3}}{R \partial \theta}-\frac{u_{1}}{R}+\theta_{1}, 2 u_{1}^{*}, 3 \theta_{1}^{*}\right] } \\
{\left[\phi_{2}, \psi_{2}, \phi_{2}^{*}\right]=} & {\left[\frac{\partial u_{3}}{\partial x}+\theta_{2}, 2 u_{2}^{*}, 3 \theta_{2}^{*}\right] . }
\end{aligned}
$$

Geometrically thick shell:

$$
\begin{aligned}
\epsilon_{1}^{z}= & \left(\epsilon_{1}+z \varkappa_{1}+z^{2} \epsilon_{1}^{*}+z^{3} \varkappa_{1}^{*}\right) /(1+z / R) \\
\epsilon_{2}^{z}= & \left(\epsilon_{2}+z \varkappa_{2}+z^{2} \epsilon_{2}^{*}+z^{3} \varkappa_{2}^{*}\right) \\
\gamma_{12}^{2}= & \left(\epsilon_{12}+z \varkappa_{12}+z^{2} \epsilon_{12}^{*}+z^{3} \varkappa_{12}^{*}\right) /(1+z / R) \\
& +\left(\epsilon_{21}+z \varkappa_{21}+z^{2} \epsilon_{21}^{*}+z^{3} \varkappa_{21}^{*}\right) \\
\gamma_{23}^{z}= & \left(\phi_{2}+z \psi_{2}+z^{2} \phi_{2}^{*}\right) \\
\gamma_{13}^{z}= & \left(\phi_{1}+z \psi_{1}+z^{2} \phi_{1}^{*}+z^{3} \psi_{1}^{*}\right) /(1+z / R),
\end{aligned}
$$

where

$$
\begin{aligned}
{\left[\epsilon_{1}, \epsilon_{1}^{*}, \chi_{1}, \chi_{1}^{*}\right]=} & {\left[\frac{\partial u_{1}}{R \partial \theta}+\frac{u_{3}}{R}, \frac{\partial u_{1}^{*}}{R \partial \theta}, \frac{\partial \theta_{1}}{R \partial \theta}, \frac{\partial \theta_{1}^{*}}{R \partial \theta}\right] } \\
{\left[\epsilon_{2}, \epsilon_{2}^{*}, \chi_{2}, \chi_{2}^{*}\right]=} & {\left[\frac{\partial u_{2}}{\partial x}, \frac{\partial u_{2}^{*}}{\partial x}, \frac{\partial \theta_{2}}{\partial x}, \frac{\partial \theta_{2}^{*}}{\partial x}\right] } \\
{\left[\epsilon_{12}, \epsilon_{12}^{*}, \chi_{12}, \chi_{12}^{*}\right]=} & {\left[\frac{\partial u_{2}}{R \partial \theta}, \frac{\partial u_{2}^{*}}{R \partial \theta}, \frac{\partial \theta_{2}}{R \partial \theta}, \frac{\partial \theta_{2}^{*}}{R \partial \theta}\right] } \\
{\left[\epsilon_{21}, \epsilon_{21}^{*}, \chi_{21}, \chi_{21}^{*}\right]=} & {\left[\frac{\partial u_{1}}{\partial x}, \frac{\partial u_{1}^{*}}{\partial x}, \frac{\partial \theta_{1}}{\partial x}, \frac{\partial \theta_{1}^{*}}{\partial x}\right] } \\
{\left[\phi_{1}, \psi_{1}, \phi_{1}^{*}, \psi_{1}^{*}\right]=} & {\left[\frac{\partial u_{3}}{R \partial \theta}-\frac{u_{1}}{R}+\theta_{1}, 2 u_{1}^{*}, \frac{u_{1}^{*}}{R}\right.} \\
& \left.+3 \theta_{1}^{*}, \frac{2 \theta_{1}^{*}}{R}\right] \\
{\left[\phi_{2}, \psi_{2}, \phi_{2}^{*}\right]=} & {\left[\frac{\partial u_{3}}{\partial x}+\theta_{2}, 2 u_{2}^{*}, 3 \theta_{2}^{*}\right] . }
\end{aligned}
$$

The constitutive relations for a typical lamina $L$ with reference to the fibre-matrix coordinate axes $\left(1^{\prime}, 2^{\prime}, 3^{\prime}\right)$ can be written as where $\left(\sigma_{1^{\prime}}, \sigma_{2^{\prime}}, \tau_{1^{\prime} 2^{\prime}}, \tau_{2^{\prime} 3^{\prime}}, \tau_{1^{\prime 3^{\prime}}}\right)$ are the stresses, and the linear strain components are given by $\left(\epsilon_{1^{\prime}}, \epsilon_{2^{\prime}}, \gamma_{12^{\prime}}, \gamma_{2^{\prime} 3^{\prime}}, \gamma_{1^{\prime} 3^{\prime}}\right)$. These are with reference to the lamina coordinates. $C_{i j} \mathrm{~s}$ are the elastic constants of the $L$ th lamina given by the following relations between these and the engineering constants:

$$
\begin{array}{ll}
C_{11}=\frac{E_{1^{\prime}}}{1-v_{1 \prime^{\prime}} v_{2^{\prime} 1^{\prime}}} ; & C_{12}=\frac{v_{1^{\prime} 2^{\prime}} E_{2^{\prime}}}{1-v_{1^{\prime} \prime^{\prime}} v_{2^{\prime} 1^{\prime}}} ; \\
C_{22}=\frac{E_{2^{\prime}}}{1-v_{1^{\prime} 2^{\prime}} \cdot v_{2^{\prime} 1^{\prime}}} ; & C_{33}=G_{12^{\prime} 2^{\prime}} ; \quad C_{44}=G_{2^{\prime} 3^{\prime}} ; \\
C_{55}=G_{1^{\prime} 3^{\prime}} .
\end{array}
$$

The stress-strain relations for the $L$ th lamina in the shell coordinates $(1,2,3)$ can be written as

$$
\sigma=\mathbf{Q} \epsilon \text {, }
$$

where

$$
\sigma=\left(\sigma_{1}, \sigma_{2}, \tau_{12}, \tau_{23}, \tau_{13}\right)^{t}
$$

and

$$
\boldsymbol{\epsilon}=\left(\epsilon_{1}, \epsilon_{2}, \gamma_{12}, \gamma_{23}, \gamma_{13}\right)^{r}
$$

are the stress and the strain vectors with respect to the shell coordinates, and, following the usual transformation rule of stresses/tensorial strains between the lamina $\left(1^{\prime}-2^{\prime}-3^{\prime}\right)$ and the laminate $(1-2-3)$ coordinate systems, the elements of the $\mathbf{Q}_{i j}$ matrix are obtained (Fig. 1). This matrix is given by the following expression:

$$
\mathbf{Q}=\left[T^{-1}\right][C]\left[T^{-1}\right]^{t}
$$

for the $L$ th lamina.

The total potential energy $\Pi$ of the system with a middle surface area $A$ enclosing a space of volume $V$ and loaded with an equivalent load vector $q$ corresponding to the nine degrees of freedom of a point on the middle surface can be written as

$$
\begin{aligned}
\Pi & =\frac{1}{2} \int_{v} \epsilon^{\prime} \sigma \mathrm{d} v-\int_{A}\left(\delta^{s}\right)^{\prime} q \mathrm{~d} A \\
& =\frac{1}{2} \int_{A}\left(\int_{\epsilon} \sigma \mathrm{d} z\right) \mathrm{d} A-\int_{A}\left(\delta^{s}\right)^{t} q \mathrm{~d} A,
\end{aligned}
$$

where

$$
\boldsymbol{\delta}^{s}=\left(u_{1}, u_{2}, u_{3}, \theta_{1}, \theta_{2}, u_{1}^{*}, u_{2}^{*}, \theta_{1}^{*}, \theta_{2}^{*}\right) .
$$

By substituting the expression for the strain components given by eqn (2) in the above expression for

$$
\left\{\begin{array}{c}
\sigma_{1^{\prime}} \\
\sigma_{2^{\prime}} \\
\tau_{1^{\prime} 2^{\prime}} \\
\tau_{2^{\prime} 3^{\prime}} \\
\tau_{1^{\prime} 3^{\prime}}
\end{array}\right\}=\left\{\begin{array}{lllll}
C_{11}^{L} & C_{12}^{L} & C_{13}^{L} & & \\
C_{21}^{L} & C_{22}^{L} & C_{23}^{L} & & \\
C_{31}^{L} & C_{32}^{L} & C_{33}^{L} & & \\
& & & C_{44}^{L} & C_{45}^{L} \\
& & & C_{54}^{L} & C_{55}^{L}
\end{array}\right\}\left\{\begin{array}{c}
\epsilon_{1^{\prime}} \\
\epsilon_{2^{\prime}} \\
\gamma_{1^{\prime} 2^{\prime}} \\
\gamma_{2^{\prime} 3^{\prime}} \\
\gamma_{1^{\prime} 3^{\prime}}
\end{array}\right\},
$$




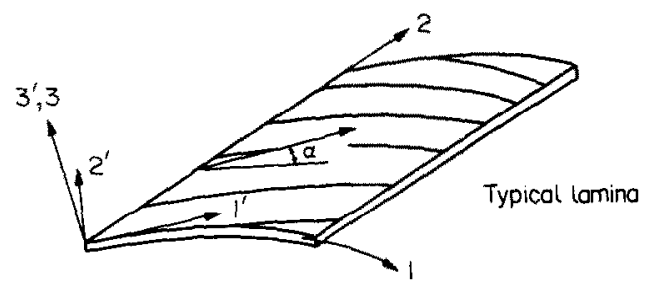

$\left(1^{\prime}, 2^{\prime}, 3^{\prime}\right)$ - Lomina reference axes

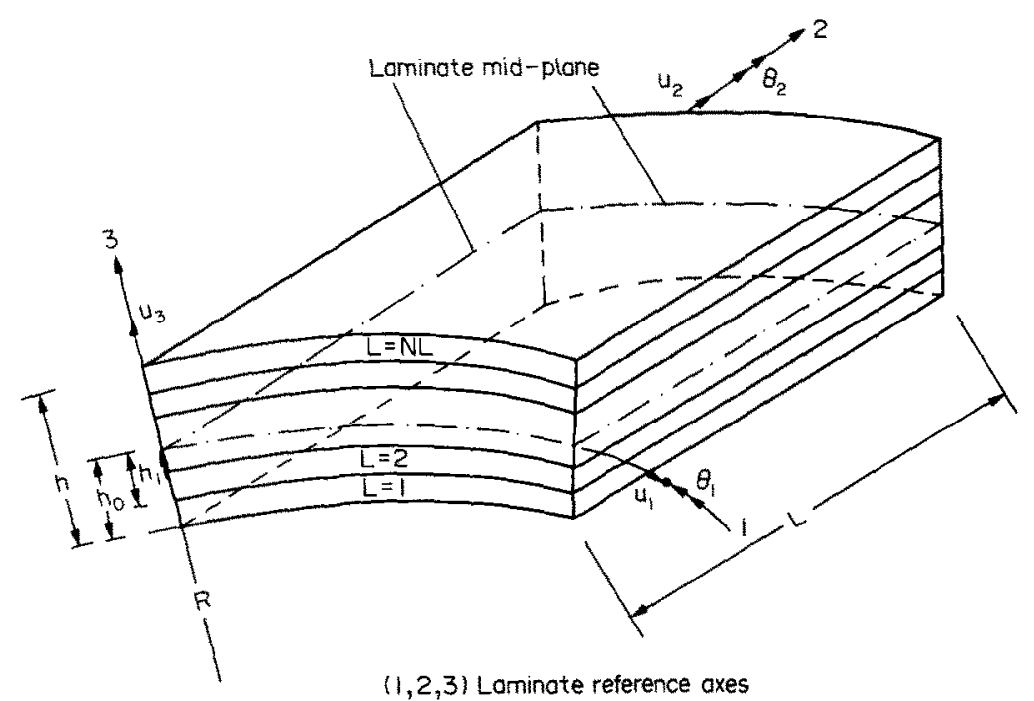

Fig. 1. Laminate geometry with positive set of lamina/laminate axis, displacement components and fibre orientation.

potential energy, the function given by eqn (4a) is then minimized while carrying out explicit integration through the shell thickness. This leads to the following 18 and 23 components of stress-resultant vector $\bar{\sigma}$ for geometrically thin and geometrically thick shell theories respectively.

Geometrically thin shell $(h / R \ll 1)$ :

$$
\left[\begin{array}{ll}
N_{1} & N_{1}^{*} \\
N_{2} & N_{2}^{*} \\
N_{12} & N_{12}^{*}
\end{array}\right]=\sum_{L=1}^{N L} \int_{h_{L}}^{h_{L+1}}\left[\begin{array}{c}
\sigma_{1} \\
\sigma_{2} \\
\tau_{12}
\end{array}\right]\left[1, z^{2}\right] \mathrm{d} z
$$

$\left[\begin{array}{cc}M_{1} & M_{1}^{*} \\ M_{2} & M_{2}^{*} \\ M_{12} & M_{12}^{*}\end{array}\right]=\sum_{L=1}^{N L} \int_{h_{L}}^{h_{L}+1}\left[\begin{array}{c}\sigma_{1} \\ \sigma_{2} \\ \tau_{12}\end{array}\right]\left[z, z^{3}\right] \mathrm{d} z$

$\left[\begin{array}{ll}Q_{2} & Q_{2}^{*} \\ Q_{1} & Q_{1}^{*}\end{array}\right]=\sum_{L=1}^{N_{L}} \int_{h_{L}}^{k_{L+1}}\left[\begin{array}{c}\tau_{23} \\ \tau_{13}\end{array}\right]\left[1, z^{2}\right] \mathrm{d} z$

$$
\left[\begin{array}{c}
S_{2} \\
S_{1}
\end{array}\right]=\sum_{L=1}^{N L} \int_{h_{L}}^{h_{L+1}}\left[\begin{array}{c}
\tau_{23} \\
\tau_{13}
\end{array}\right] z \mathrm{~d} z
$$

$$
\begin{aligned}
& {\left[\begin{array}{ll}
N_{1} & N_{1}^{*} \\
N_{2} & N_{2}^{*} \\
N_{12} & N_{12}^{*} \\
N_{21} & N_{21}^{*}
\end{array}\right]=\sum_{L=1}^{N L} \int_{h_{L}}^{h_{L}+1}\left[\begin{array}{cccc}
1 & 0 & 0 & 0 \\
0 & A & 0 & 0 \\
0 & 0 & 1 & 0 \\
0 & 0 & 0 & A
\end{array}\right]\left[\begin{array}{c}
\sigma_{1} \\
\sigma_{2} \\
\tau_{12} \\
\tau_{21}
\end{array}\right]\left[1, z^{2}\right] \mathrm{d} z} \\
& {\left[\begin{array}{ll}
M_{1} & M_{1}^{*} \\
M_{2} & M_{2}^{*} \\
M_{12} & M_{12}^{*} \\
M_{21} & M_{21}^{*}
\end{array}\right]=\sum_{L=1}^{N L} \int_{h_{L}}^{h_{L}+1}\left[\begin{array}{cccc}
1 & 0 & 0 & 0 \\
0 & A & 0 & 0 \\
0 & 0 & 1 & 0 \\
0 & 0 & 0 & A
\end{array}\right] \quad\left[\begin{array}{c}
\sigma_{1} \\
\sigma_{2} \\
\tau_{12} \\
\tau_{21}
\end{array}\right]\left[z, z^{3}\right] \mathrm{d} z}
\end{aligned}
$$




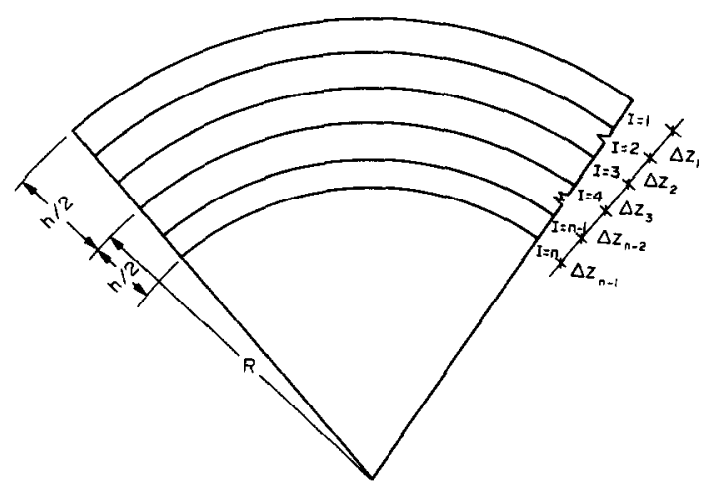

Fig. 2. A typical cross-section of shell showing unequal spacing of pivot points.

$\left[\begin{array}{ll}Q_{2} & Q_{2}^{*} \\ Q_{1} & Q_{1}^{*}\end{array}\right]=\sum_{L=1}^{N L} \int_{h_{L}}^{h_{L}+1}\left[\begin{array}{ll}A & 0 \\ 0 & 1\end{array}\right]\left[\begin{array}{l}\tau_{23} \\ \tau_{13}\end{array}\right]\left[1, z^{2}\right] \mathrm{d} z$

$$
S_{2}=\sum_{L=1}^{N L} \int_{h_{L}}^{h_{L+1}} \tau_{23} A z \mathrm{~d} z
$$$$
\left[S_{1} S_{1}^{*}\right]=\sum_{L=1}^{N L} \int_{h_{L}}^{h_{L}+1} \tau_{13}\left[z, z^{3}\right] \mathrm{d} z
$$

where $A=(1+z / R)$ and $N L$ is the number of layers.

Upon integration, these expressions are rewritten in matrix form as

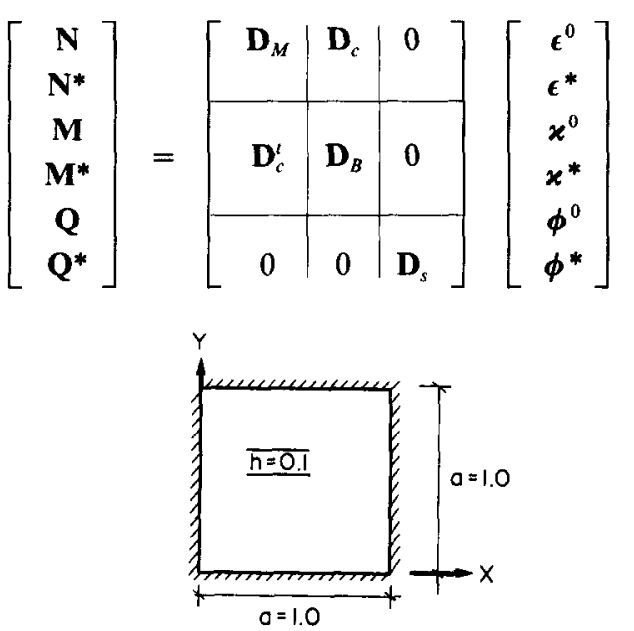

Fig. 3a. Fixed, two-layered square laminated plate under uniform transverse pressure. or

in which

$$
\bar{\sigma}=\mathbf{D} \bar{\epsilon},
$$

$$
\begin{aligned}
\mathbf{N}=\left(N_{1}, N_{2}, N_{12}\right)^{t} ; & \mathbf{N}^{*}=\left(N_{1}^{*}, N_{2}^{*}, N_{12}^{*}\right)^{t} \\
\mathbf{M}=\left(M_{1}, M_{2}, M_{12}\right)^{t ;} & \mathbf{M}^{*}=\left(M_{1}^{*}, M_{2}^{*}, M_{12}^{*}\right)^{t} \\
\mathbf{Q}=\left(Q_{2}, Q_{1}\right)^{t} ; \quad \mathbf{Q}^{*} & =\left(Q_{2}^{*}, Q_{1}^{*}, S_{2}, S_{1}\right)^{t}
\end{aligned}
$$

$\epsilon^{0}=\left(\epsilon_{1}, \epsilon_{2}, \epsilon_{12}\right)^{t}$

$$
\epsilon^{*}=\left(\epsilon_{1}^{*}, \epsilon_{2}^{*}, \epsilon_{12}^{*}\right)^{t}
$$

$x^{0}=\left(x_{1}, x_{2}, x_{12}\right)^{t}$

$$
x^{*}=\left(x_{1}^{*}, x_{2}^{*}, x_{12}^{*}\right)^{t}
$$

$\phi^{0}=\left(\phi_{2}, \phi_{1}\right)^{t}$

$\phi^{*}=\left(\phi_{2}^{*}, \phi_{1}^{*}, \psi_{2}, \psi_{1}\right)^{\prime}$

for the geometrically thin shell theory, and

$$
\begin{aligned}
& \mathbf{N}=\left(N_{1}, N_{2}, N_{12}, N_{21}\right)^{t} ; \mathbf{N}^{*}=\left(N_{1}^{*}, N_{2}^{*}, N_{12}^{*}, N_{21}^{*}\right)^{t} \\
& \mathbf{M}=\left(M_{1}, M_{2}, M_{12}, M_{21}\right)^{t} ; \\
& \mathbf{M}^{*}=\left(M_{1}^{*}, M_{2}^{*}, M_{12}^{*}, M_{21}^{*}\right)^{t} \\
& \mathbf{Q}=\left(Q_{2}, Q_{1}\right)^{*} ; \quad \mathbf{Q}^{*}=\left(Q_{2}^{*}, Q_{1}^{*}, S_{2}, S_{1}, S_{1}^{*}\right)^{t}
\end{aligned}
$$

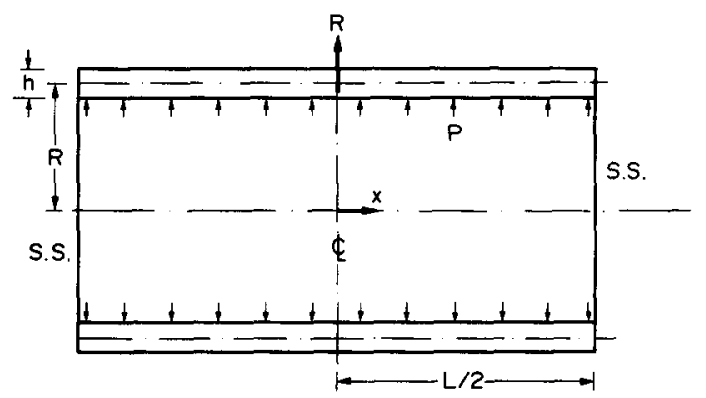

Fig. 3c. Simply supported cylindrical shell subjected to uniform internal pressure.

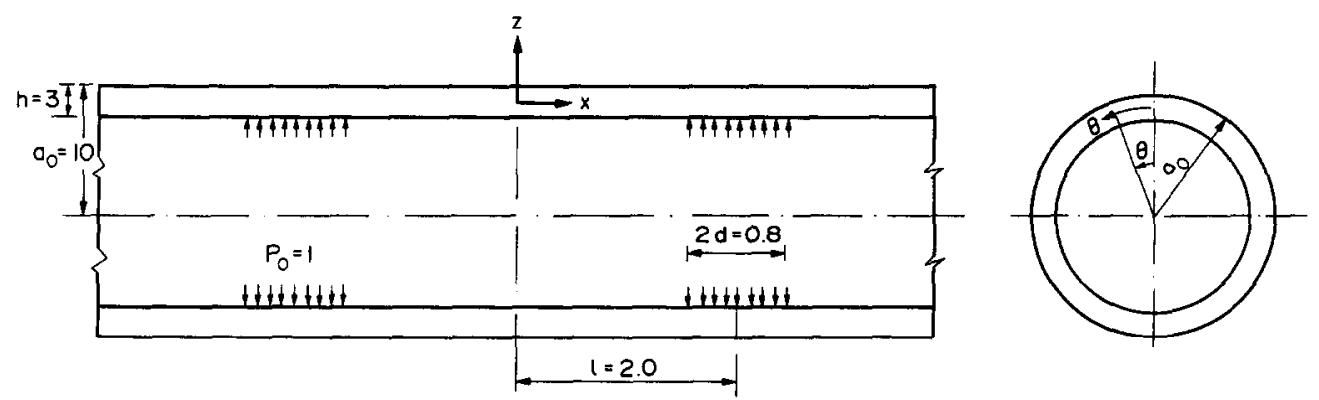

Fig. 3b. Long circular cylindrical shell subjected to periodically spaced band loads. 


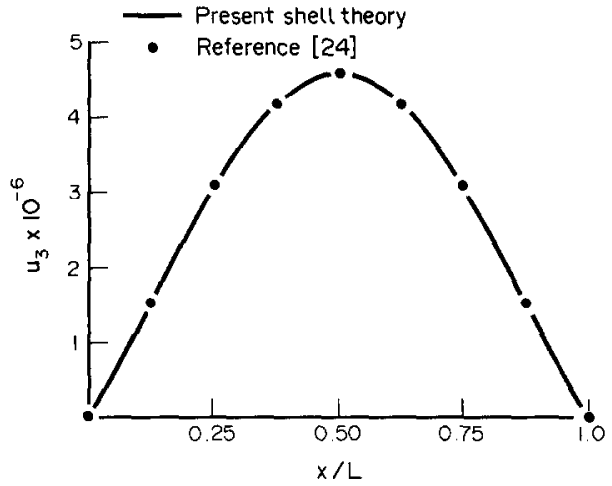

Fig. 4a. Variation of $U_{3}$ along the length at $Y=a / 2$.

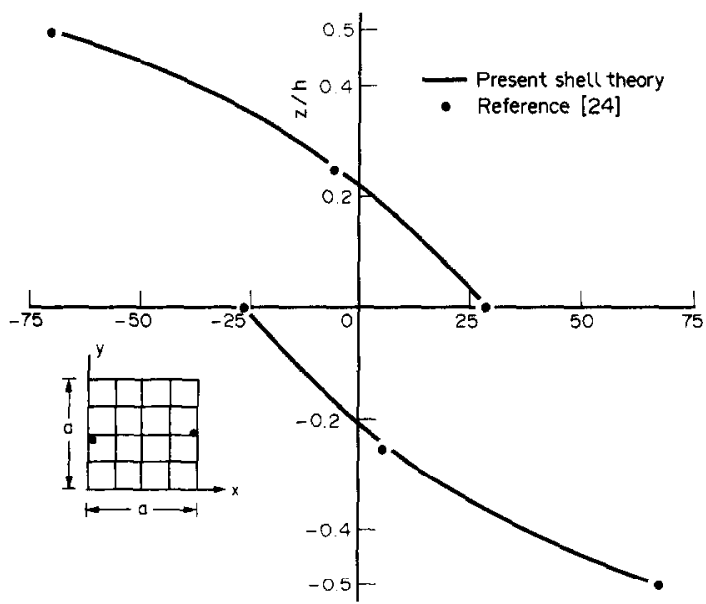

Fig. 4b. Distribution of a $\sigma_{1}$ across the thickness at $X=0$, $Y=a / 2$.

$$
\begin{aligned}
& \epsilon^{0}=\left(\epsilon_{1}, \epsilon_{2}, \epsilon_{12}, \epsilon_{21}\right)^{t} ; \epsilon^{*}=\left(\epsilon_{1}^{*}, \epsilon_{2}^{*}, \epsilon_{12}^{*}, \epsilon_{21}^{*}\right)^{t} \\
& x^{0}=\left(\varkappa_{1}, x_{2}, \varkappa_{12}, \varkappa_{21}\right)^{t} ; x^{*}=\left(x_{1}^{*}, x_{2}^{*}, x_{12}^{*}, x_{21}^{*}\right)^{t} \\
& \phi^{0}=\left(\phi_{2}, \phi_{1}\right)^{t ;} \quad \phi^{*}=\left(\phi_{2}^{*}, \phi_{1}^{*}, \psi_{2}, \psi_{1}, \psi_{1}^{*}\right)^{t}
\end{aligned}
$$

for the geometrically thick shell theory. The individual sub-matrices of the rigidity matrix $\mathbf{D}$ are

$$
\begin{aligned}
& \mathbf{D}_{M}-\text { membrane rigidity matrix; } \\
& \mathbf{D}_{c}-\text { membrane-flexure coupling matrix; } \\
& \mathbf{D}_{B}-\text { flexure rigidity matrix; and } \\
& \mathbf{D}_{s}-\text { shear rigidity matrix. }
\end{aligned}
$$

The elements in each of these sub-matrices are defined in Appendix A and Appendix B for the geometrically thin and thick shell theories respectively.

Having obtained the displacements, strains are determined by eqn (2) and the stresses via the constitutive equation [eqn (3b)]. The interlaminar stresses $\left(\tau_{23}, \tau_{13}\right)$ cannot be accurately estimated by eqn ( $\left.3 \mathrm{~b}\right)$. This is mainly because the interlaminar stresses have

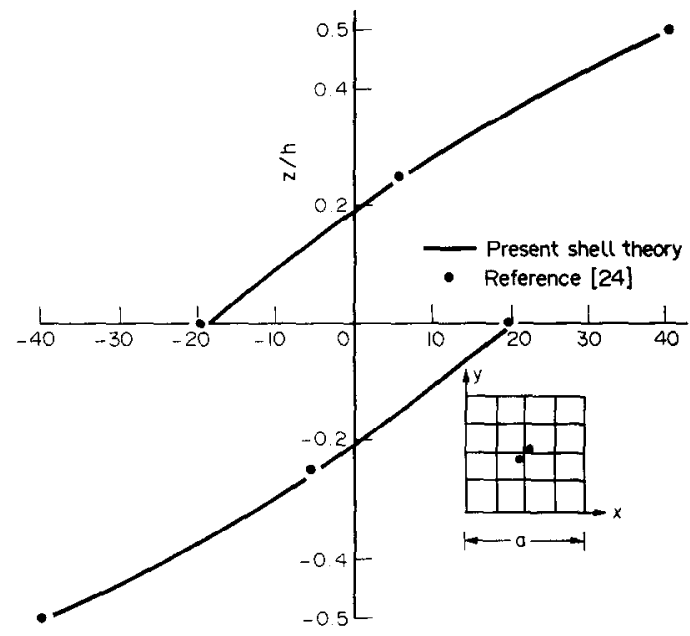

Fig. $4 \mathrm{c}$. Distribution of $\sigma_{1}$ across the thickness at the centre.

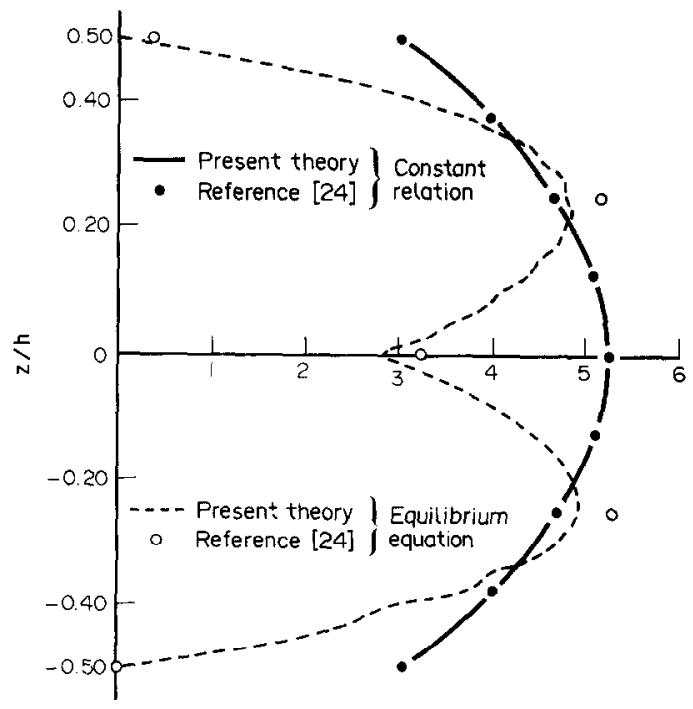

Fig. 4d. Variation of $\tau_{13}$ across the thickness at $X=0$, $Y=a / 2$.

to maintain continuity across the interfaces whereas they are discontinuous when evaluated by constitutive law. A three-dimensional analysis becomes very complex. Even three-dimensional displacement-based finite element schemes will suffer from the same defect of interlaminar stress discontinuity across the interfaces. For these reasons, a better accuracy for interlaminar stresses between layers $L$ and $L+1$ at $z=h_{L+1}$ may be attained by intcgrating the equilibrium equation of elasticity for each layer over the lamina thickness and summing over layers 1 to $L$ in the transverse directions.

The equation of equilibrium representing the pointwise equilibrium can be written as

$$
\begin{aligned}
& \frac{\partial \sigma_{1}}{A R \partial \theta}+\frac{\partial \tau_{12}}{\partial x}+\frac{\partial \tau_{13}}{\partial z}+\frac{2}{A R} \tau_{13}=0 \\
& \frac{\partial \tau_{12}}{A R \partial \theta}+\frac{\partial \sigma_{2}}{\partial x}+\frac{\partial \tau_{23}}{\partial z}+\frac{1}{A R} \tau_{23}=0
\end{aligned}
$$




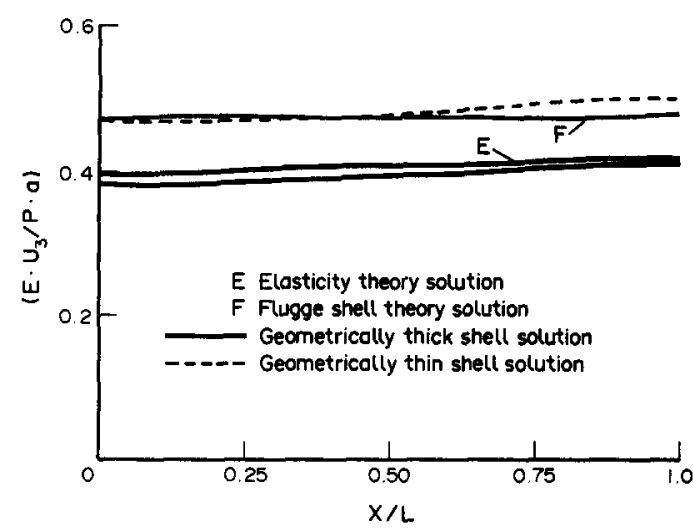

Fig. 5a. Variation of $U_{3}$ along the length.

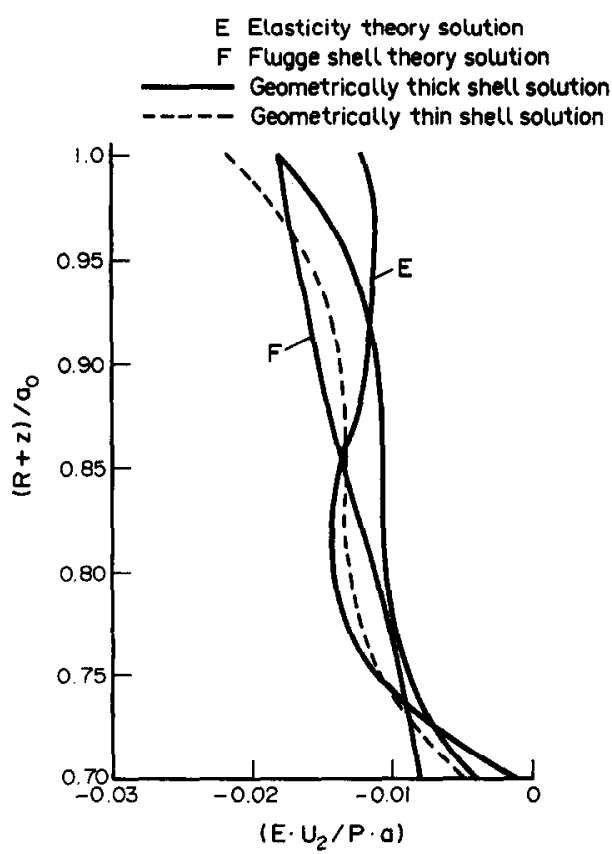

Fig. 5b. Variation of $U_{2}$ across the thickness at $x=0.8$.

where $A=1$ for the geometrically thin shell theory and $A=(1+z / R)$ for the geometrically thick shell theory. In the above equations, the body forces are assumed to be negligible.

\section{FINITE ELEMENT FORMULATION}

For the present study, a nine-noded quadrilateral (Lagrangian family) two-dimensional $C^{0}$ continuous isoparametric element with nine degrees of freedom per node is developed. The displacement vector $\boldsymbol{\delta}^{s}$ at any point on the reference surface is given by

$$
\delta^{s}=\sum_{i=1}^{N N} N_{i}(\theta, x) \delta_{i}^{s},
$$

where $\delta_{i}^{s}$ is the displacement vector corresponding to node $i, N_{i}$ is the interpolating or shape function associated with node $i$, and $N N$ is the total number of nodes per element (nine in this case).

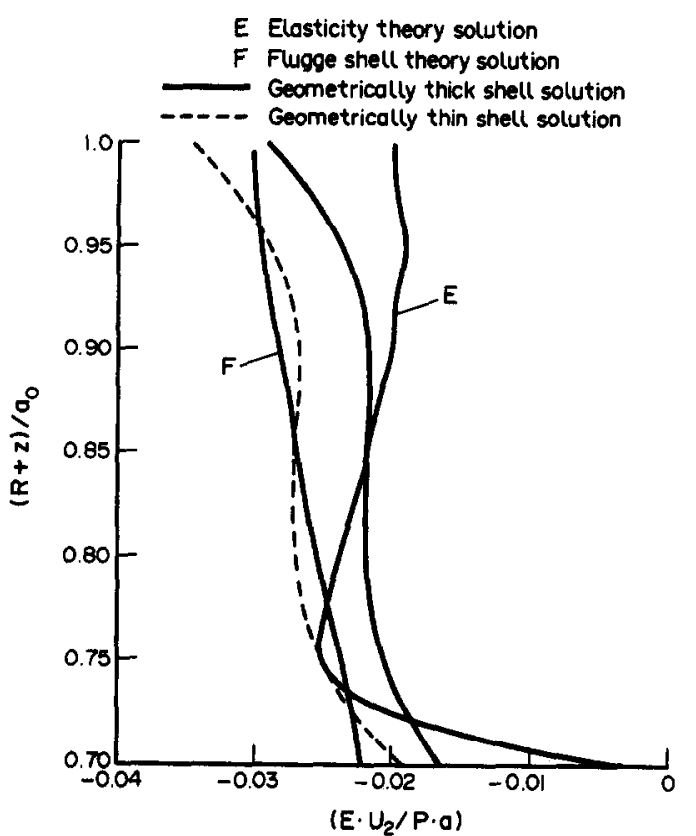

Fig. Sc. Variation of $U_{2}$ across the thickness at $x=1.6$.

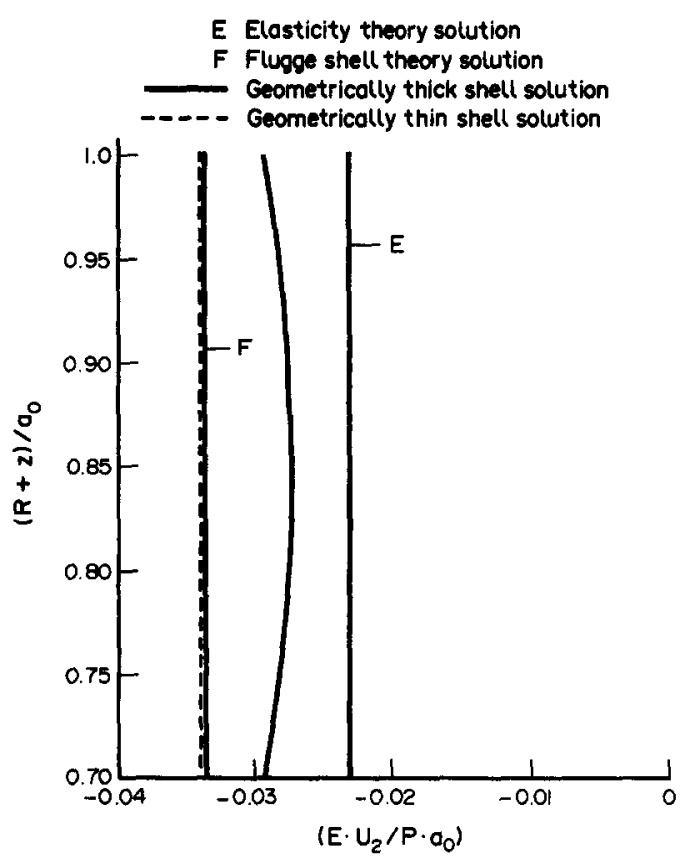

Fig. 5d. Variation of $U_{2}$ across the thickness at $x=2.0$.

Knowing the generalized displacement vector $\delta^{s}$ at all points within the element, the generalized midsurface strains at any point given by eqns (2b) and (2d) for a geometrically thin and a thick shell respectively can be expressed in terms of nodal displacements in matrix form as follows:

$$
\bar{\epsilon}=\sum_{i=1}^{N N} B_{i} \delta_{i}^{s}
$$

where $B_{i}$ is a differential operator matrix [22] of shape functions. 


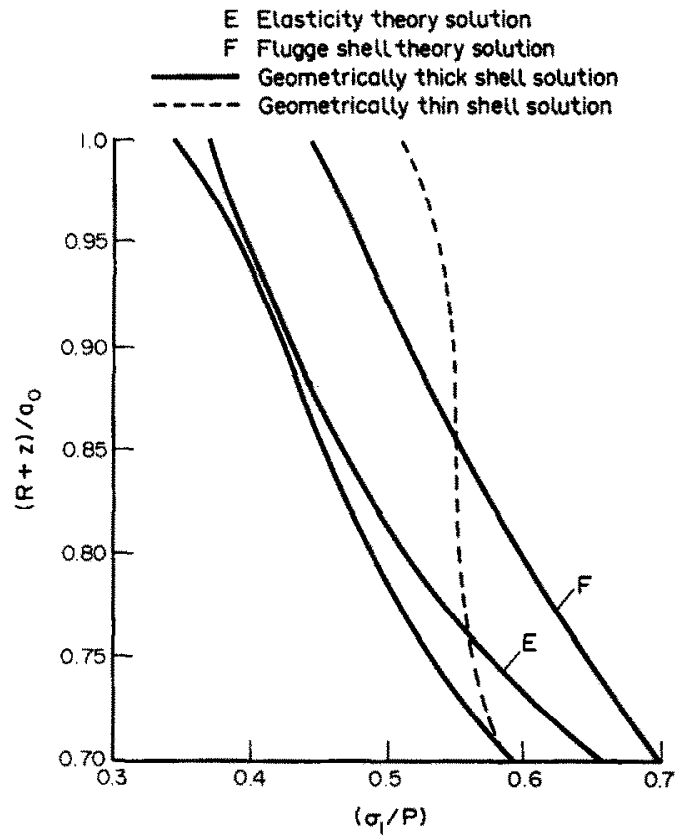

Fig. Se. Variation of $\sigma_{1}$ across the thickness at $x=0.0$.

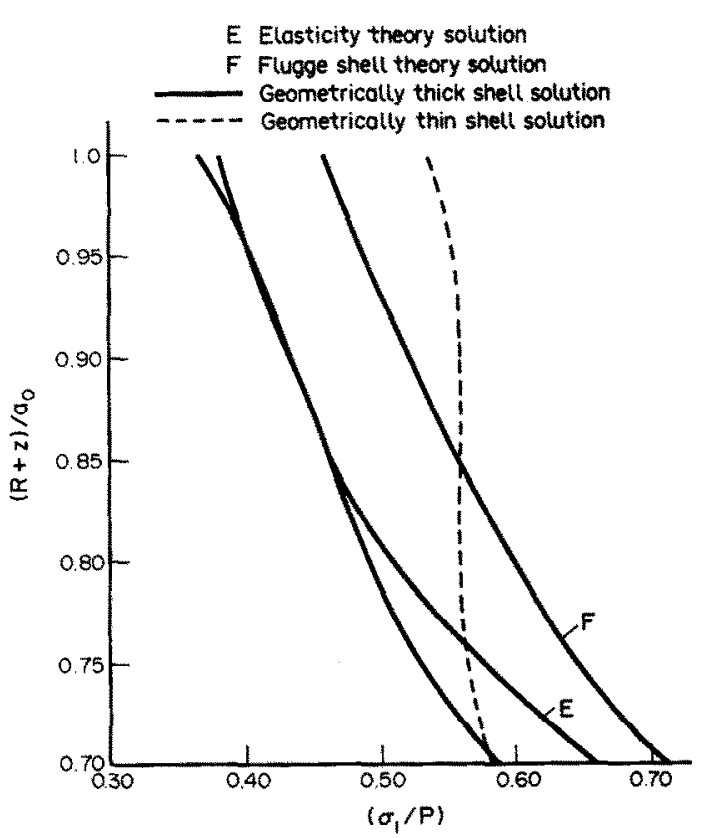

Fig. 5f. Variation of $\sigma_{1}$ across the thickness at $x=0.8$.

Using the standard finite element technique, the total domain is discretized into $N E$ sub-domains or elements such that

$$
\Pi(\boldsymbol{\delta})=\sum_{e=1}^{N E} \Pi^{e}\left(\boldsymbol{\delta}^{s}\right),
$$

where $\Pi$ and $\Pi^{e}$ are the potential energies of the structure and the element respectively. We further have

$$
\Pi^{e}\left(\delta^{s}\right)=U^{e}-W^{e}
$$

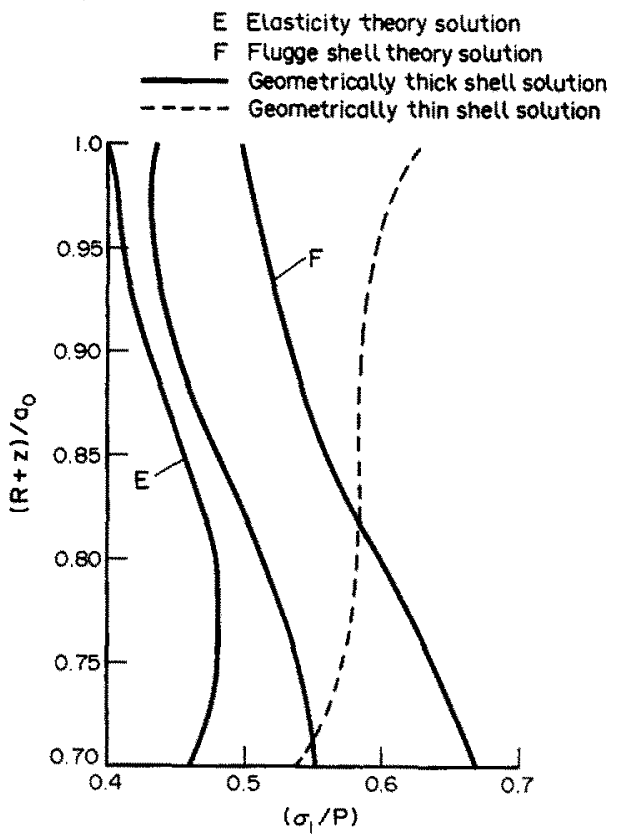

Fig. 5g. Variation of $\sigma_{1}$ across the thickness at $x=1.6$.

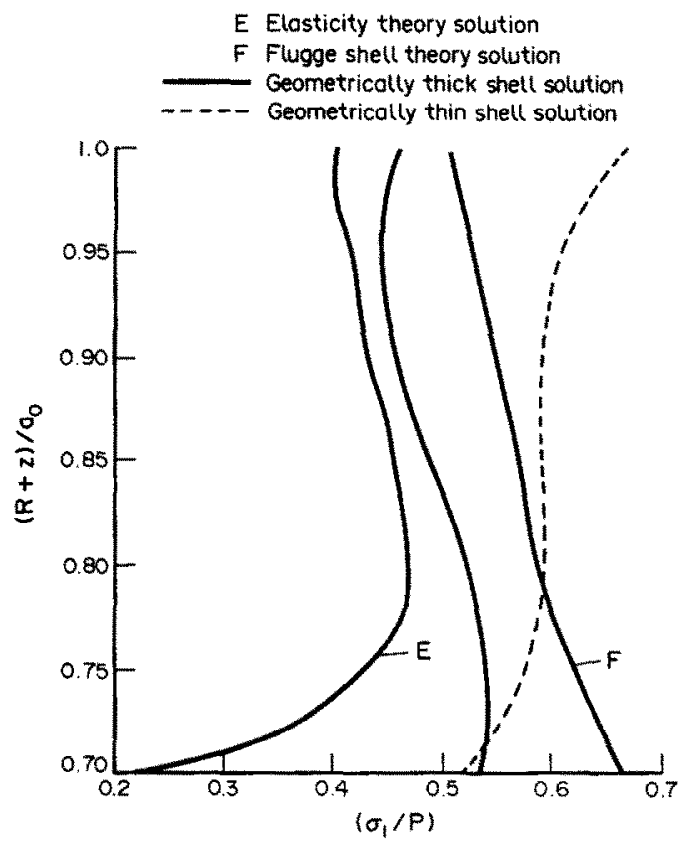

Fig. 5h. Variation of $\sigma_{1}$ across the thickness at $x=2.0$.

in which $U^{e}$ and $W^{e}$ are the internal strain energy and external work done respectively for an element.

Based on the principle of minimum potential energy for the structure and by evaluating the $D$ and $\mathbf{B}_{i}$ matrices as given by eqns (6a) and (9) respectively, the element stiffness matrix can be obtained by using the standard relation

$$
\mathbf{K}_{i j}^{e}=\int_{-1}^{1} \int_{-1}^{1} \mathbf{B}_{i}^{t} \mathbf{D} \mathbf{B}_{i}|J| \partial \xi \partial \eta .
$$




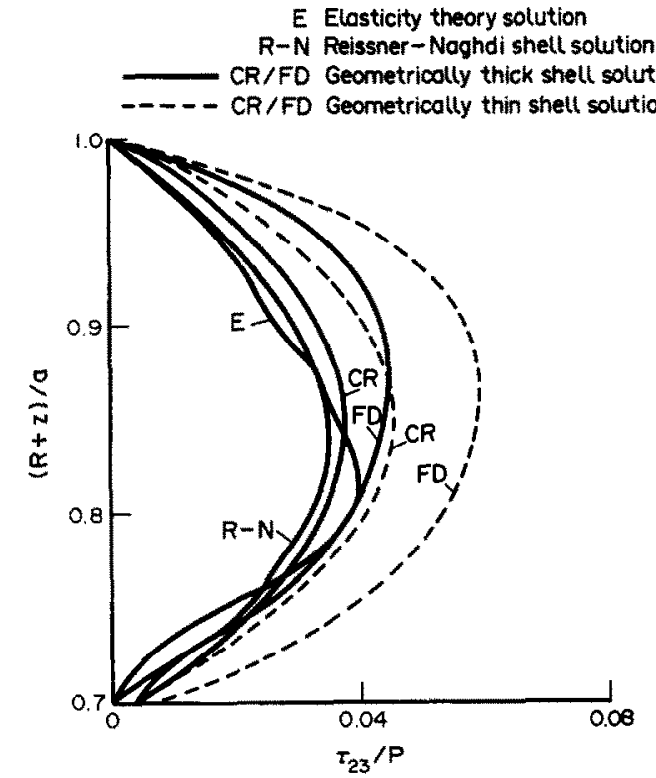

Fig. 5i. Variation of $\tau_{23}$ across the thickness at $x=0.4$.

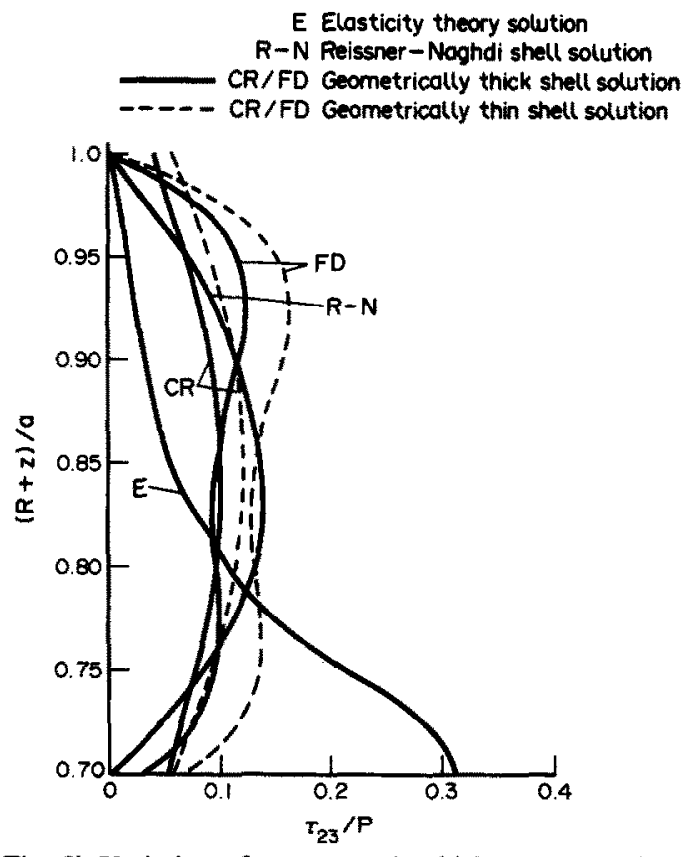

Fig. 5j. Variation of $\tau_{23}$ across the thickness at $x=1.6$.

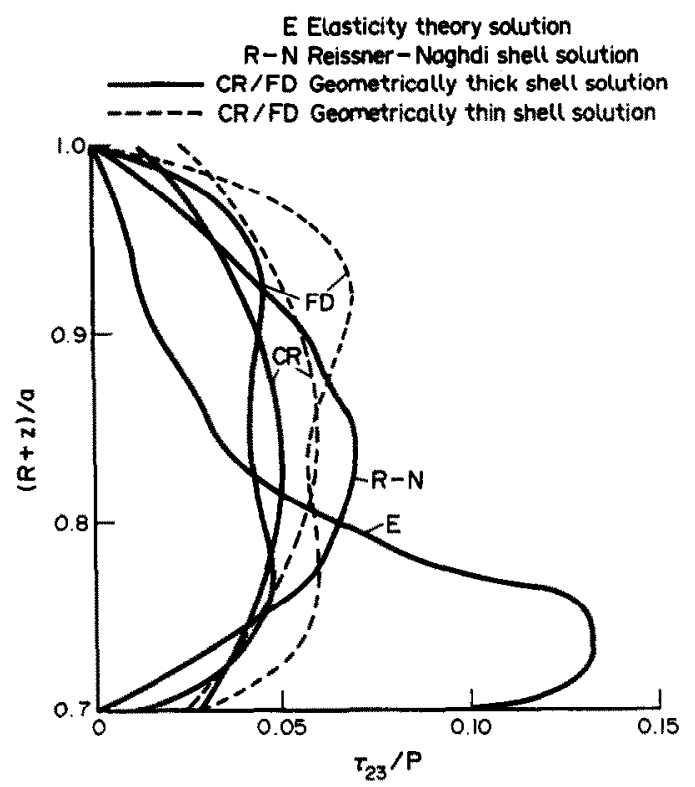

Fig. 5k. Variation of $\tau_{23}$ across the thickness at $x=1.8$.

Similarly, the distributed pressure loading on an element is easily transformed into equivalent nodal loads using the virtual work principle. Thus the consistent load vector $\boldsymbol{P}_{i}$ due to a uniformly distributed transverse load $q$ can be written as

$$
\mathbf{P}_{i}^{e}=\int_{-1}^{1} \int_{-1}^{1} N_{i}^{t} q|J| \partial \xi \partial \eta, i=1, N N
$$

where $|J|$ is the determinant of the standard Jacobian matrix.
Knowing the element stiffness and the load matrix, they are assembled to represent a particular geometry with prescribed boundary conditions. The governing equations are then solved to obtain a discrete set of displacements. Having obtained a set of nodal displacements, strains within a particular element are determined through eqn (2) and then the in-plane stresses are determined from eqn (3b) at the desired locations. The transverse shear stresses thus obtained from the constitutive relations are found to be discontinuous at the interfaces. Thus, as previously discussed, a better approach is to use 


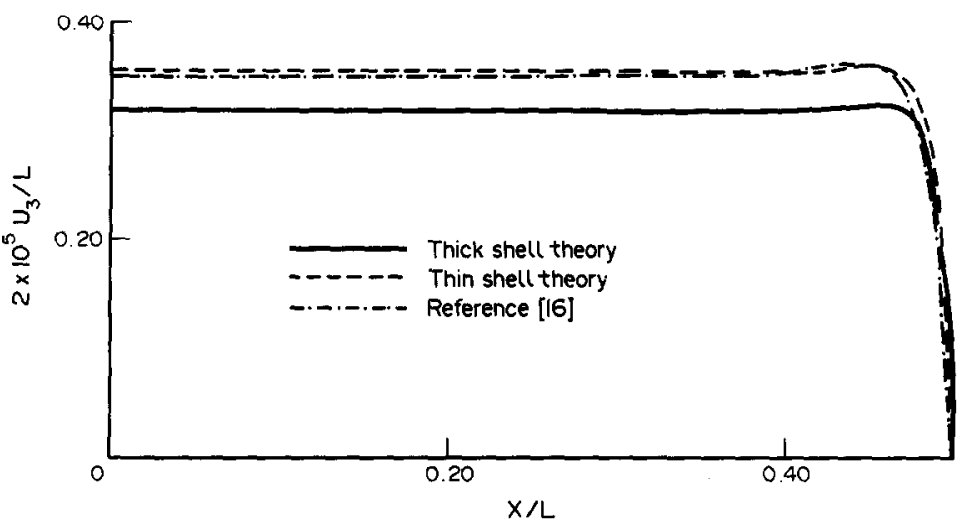

Fig. 6a. Variation of $U_{3}$ along the length for $\left(90^{\circ} / 0^{\circ} / 90^{\circ}\right)$ lamination.

the equilibrium equations for determination of the transverse shear stresses. Equation (7) is rewritten as

$$
\begin{aligned}
& \frac{\partial \tau_{13}}{\partial z}+\frac{2}{A R} \tau_{13}=-\left(\frac{\partial \sigma_{1}}{A R \partial \theta}+\frac{\partial \tau_{12}}{\partial X}\right) \\
& \frac{\partial \tau_{23}}{\partial z}+\frac{1}{A R} \tau_{23}=-\left(\frac{\partial \tau_{12}}{A R \partial \theta}+\frac{\partial \sigma_{2}}{\partial X}\right) .
\end{aligned}
$$

Thus having obtained the in-plane stresses $\left(\sigma_{1}, \sigma_{2}, \tau_{12}\right)$ in the laminae, the transverse shear stresses are determined on the basis of in-plane stress variations.

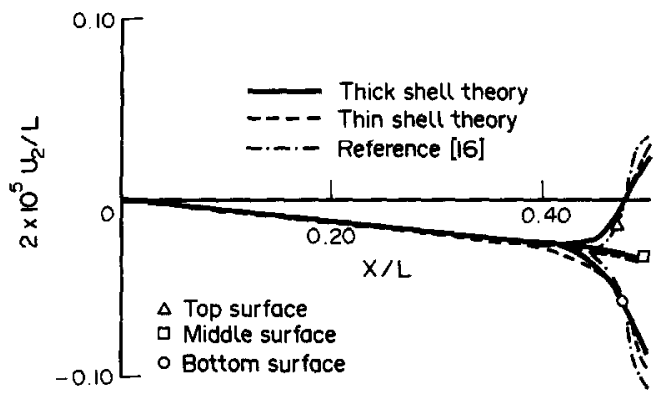

Fig. 6b. Variation of $U_{2}$ along the length for $\left(90^{\circ} / 0^{\circ} / 90^{\circ}\right)$ lamination.
As solution of the equilibrium equations is a vital component in the overall solution strategy, the numerical methodology used in solving these equations is discussed in detail. The solution procedures are described.

The in-plane stresses $\left(\sigma_{1}, \sigma_{2}, \tau_{12}\right)$ at different points in the thickness direction of a particular element are determined at all nine Gauss points, through solution of the constitutive equations. Having obtained the in-plane stresses acting on any surface of a particular element at all nine Gauss points, the variations of these stresses on the surface of the element are expressed using a polynomial in $\theta$ and $x$ :

$$
\begin{aligned}
\sigma_{1}(z)= & C_{1}^{1}+C_{2}^{1} R \theta+C_{3}^{1} x+C_{4}^{1}(R \theta)^{2}+C_{5}^{1}(R \theta) x \\
& +C_{6}^{1} x^{2}+C_{7}^{1}(R \theta)^{2} x+C_{8}^{1}(R \theta) x^{2} \\
& +C_{9}^{1}(R \theta)^{2} x^{2} . \\
\sigma_{2}(z)= & C_{1}^{2}+C_{2}^{2} R \theta+C_{3}^{2} x+C_{4}^{2}(R \theta)^{2}+C_{5}^{2}(R \theta) x \\
& +C_{6}^{2} x^{2}+C_{7}^{2}(R \theta)^{2} x+C_{8}^{2}(R \theta) x^{2} \\
& +C_{9}^{2}(R \theta)^{2} x^{2} . \\
\tau_{12}(z)= & C_{1}^{3}+C_{2}^{3} R \theta+C_{3}^{3} x+C_{4}^{3}(R \theta)^{2}+C_{5}^{3}(R \theta) x \\
& +C_{6}^{3} x^{2}+C_{7}^{3}(R \theta)^{2} x+C_{8}^{3}(R \theta) x^{2} \\
& +C_{9}^{3}(R \theta)^{2} x^{2},
\end{aligned}
$$

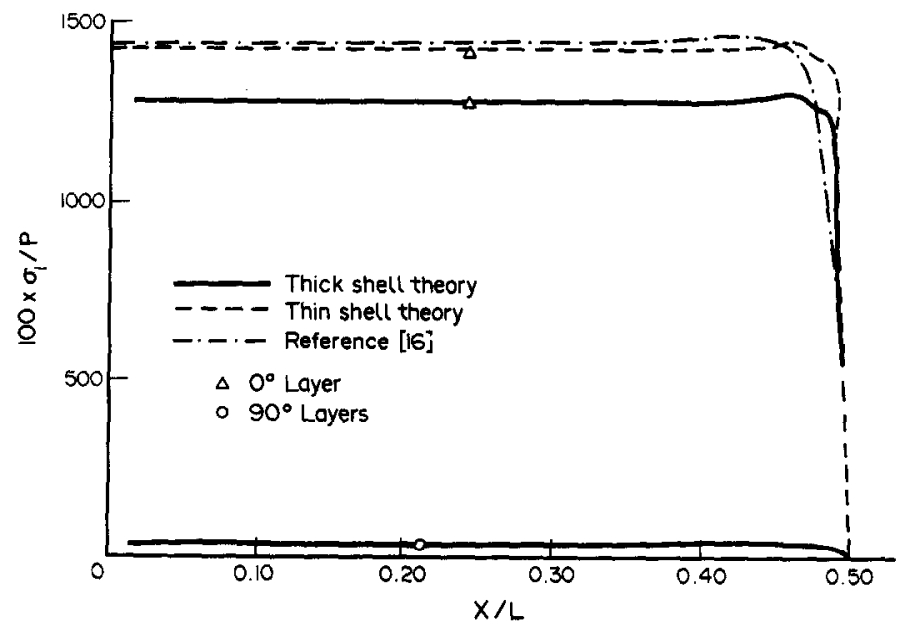

Fig. $6 \mathrm{c}$. Variation of $\sigma_{1}$ along the length for $\left(90^{\circ} / 0^{\circ} / 90^{\circ}\right)$ lamination. 


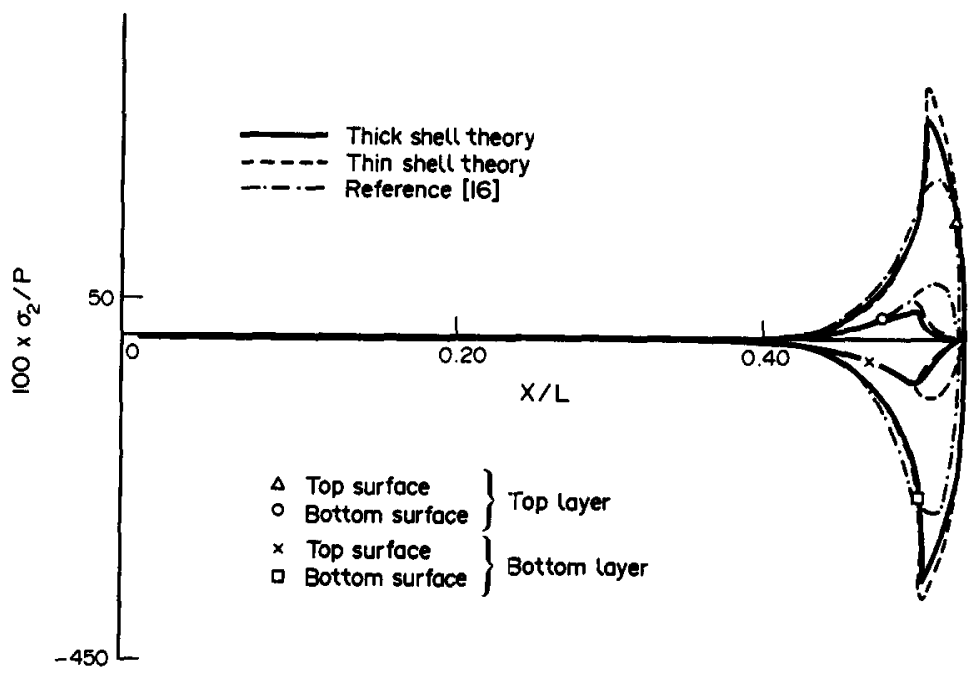

Fig. 6d. Variation of $\sigma_{2}$ along the length for $\left(90^{\circ} / 0^{\circ} / 90^{\circ}\right)$ lamination.

where the constants $C_{1}^{i}$ to $C_{9}^{i}(i=1,3)$ are evaluated knowing the values of the stresses and their positions at all nine Gauss points for different points in the thickness directions. Thus, by substituting these constants in eqn (15), the stress fields acting on different surfaces of each layer are known. From these stress fields, the derivatives of the stresses with respect to $\theta$ and $x$ are computed from the following expressions:

$$
\begin{aligned}
& \frac{\partial \sigma_{1}(z)}{R \partial \theta}=C_{2}^{1}+2 C_{4}^{1} R \theta+C_{5}^{1} x \\
& +2 C_{7}^{1}(R \theta) x+C_{8}^{1} x^{2}+2 C_{9}^{1}(R \theta) x^{2} \\
& \frac{\partial \sigma_{2}(z)}{\partial x}=C_{3}^{2}+C_{5}^{2} R \theta+2 C_{6}^{2} x \\
& +C_{7}^{2}(R \theta)^{2}+2 C_{8}^{2}(R \theta) x+2 C_{9}^{2}(R \theta)^{2} x \\
& \frac{\partial \tau_{12}(z)}{R \partial \theta}=C_{2}^{3}+2 C_{4}^{3} R \theta+C_{5}^{3} x \\
& +2 C_{7}^{3}(R \theta) x+C_{8}^{3} x^{2}+2 C_{9}^{3}(R \theta) x^{2} \\
& \frac{\partial \tau_{12}(z)}{\partial x}=C_{3}^{3}+C_{5}^{3} R \theta+2 C_{6}^{3} x \\
& +C_{7}^{3}(R \theta)^{2}+2 C_{8}^{3}(R \theta) x+2 C_{9}^{3}(R \theta)^{2} x .
\end{aligned}
$$

In this study, the values of derivatives are evaluated at four Gauss points corresponding to the $2 \times 2$ Gauss quadrature rule for shear. Once the derivatives of the in-plane stresses with respect to $\theta$ and $x$ are known at a particular Gauss point, say at the top, centre and bottom of various layers [thus evaluating the right-hand side of the equilibrium equation, eqn (14)], through-the-thickness shear stress variations are evaluated using the finite difference technique.

In this work, two well-known finite difference techniques, (a) the forward difference method and (b) the central difference method, are used to derive the shear stresses at different points in the lamina. Thus eqn (14) can be written in numerical form as follows (see Fig. 2).

\section{Forward difference technique}

$$
\begin{gathered}
\begin{array}{c}
\tau_{13(I+1)}-\tau_{13(I)} \\
\Delta z_{I}
\end{array}+\frac{2}{A_{(I)} R} \tau_{13(I)}=-\left(\frac{\partial \sigma_{1}}{A R \partial \theta}+\frac{\partial \tau_{12}}{\partial X}\right)_{(I)} \\
\begin{array}{c}
\therefore \tau_{13(I+1)}=\Delta z_{I}\left(-\frac{\partial \sigma_{1}}{A R \partial \theta}-\frac{\partial \tau_{12}}{\partial X}-\frac{2}{A R} \tau_{13}\right)_{(I)} \\
+\tau_{13(I)}
\end{array} \\
\frac{\tau_{23(I+1)}-\tau_{23(I)}}{\Delta z_{I}}+\frac{\tau_{23(I)}}{A_{(I)} R}=-\left(\frac{\partial \tau_{12}}{A R \partial \theta}+\frac{\partial \sigma_{2}}{\partial X}\right)_{(I)} \\
\therefore \tau_{23(I+1)}=\Delta z_{I}\left(-\frac{\partial \tau_{12}}{A R \partial \theta}-\frac{\partial \sigma_{2}}{\partial X}-\frac{\tau_{23}}{A R}\right)_{(I)} \\
+\tau_{23(I)} .
\end{gathered}
$$

Assuming that the laminate has shear free surfaces, we have $\tau_{13(1)}$ and $\tau_{23(1)}=0$. With these as the starting values, the values of the transverse shear stresses are evaluated at all the other points through the thickness. The other free surface conditions are not utilized, although they are available, because we are dealing with first-order differential equations in $\tau_{23}$ and $\tau_{13}$, which need only one initial condition. This is a physical paradox.

\section{Central difference technique}

$$
\frac{\tau_{13(I+1)}-\left(1-\alpha^{2}\right) \tau_{13(l)}-\alpha^{2} \tau_{13(I-1)}}{\alpha(\alpha+1) \Delta z_{I}}+\frac{2}{A_{(I)} R} \tau_{13(l)}=P_{(l)}
$$

where

$$
\begin{gathered}
P_{(I)}=-\left(\frac{\partial \sigma_{1}}{A R \partial \theta}+\frac{\partial \tau_{12}}{\partial X}\right)_{(I)} \\
\therefore \tau_{13(I+1)}=\alpha(\alpha+1) \Delta z_{1}\left(P-\frac{2}{A R} \tau_{13}\right)_{(I)} \\
+\left(1-\alpha^{2}\right) \tau_{13(I)}+\alpha^{2} \tau_{13(I-1)}
\end{gathered}
$$




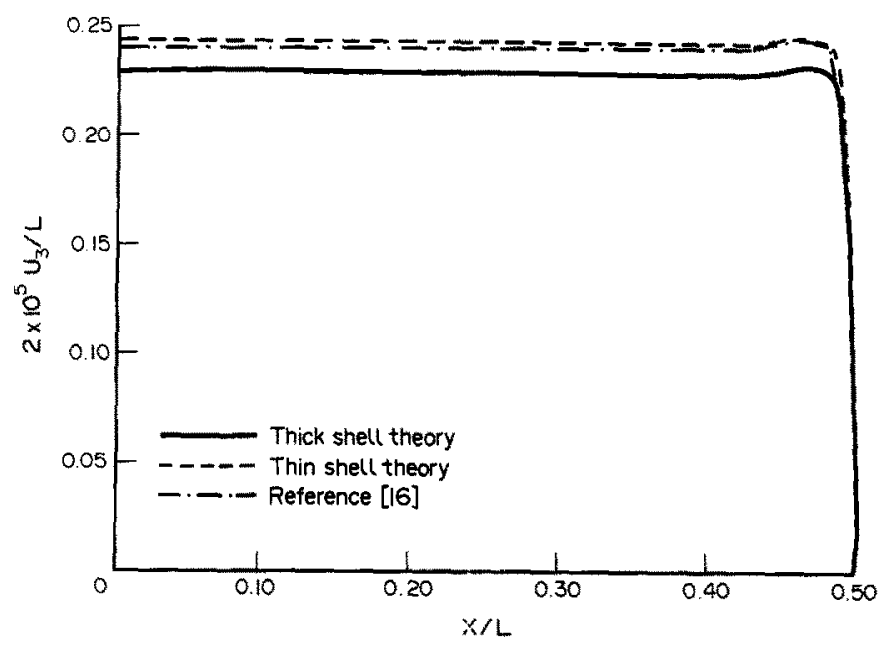

Fig. 7a. Variation of $U_{3}$ along the length for $\left(0^{\circ} / 90^{\circ}\right)$ lamination.

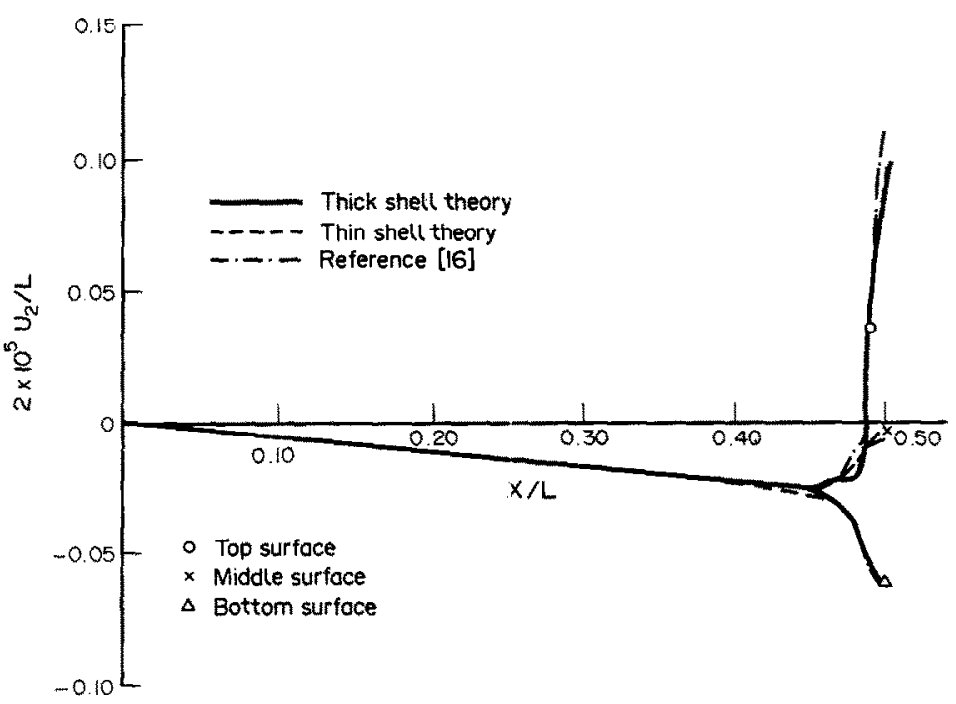

Fig. $7 \mathrm{~b}$. Variation of $U_{2}$ along the length for $\left(0^{\circ} / 90^{\circ}\right)$ lamination.

$$
\begin{aligned}
& \frac{\tau_{23(I+1)}-\left(1-\alpha^{2}\right) \tau_{23(I)}-\alpha^{2} \tau_{23(I-1)}}{\alpha(\alpha+1) \Delta z_{1}} \\
& +\frac{1}{A_{(I)} R} \tau_{23(I)}=Q_{(I)}
\end{aligned}
$$

where

$$
\begin{gathered}
Q_{(l)}=-\left(\frac{\partial \tau_{12}}{A R \partial \theta}+\frac{\partial \sigma_{2}}{\partial X}\right)_{(l)} \\
\therefore \tau_{23(I+1)}=\alpha(\alpha+1) \Delta z_{I}\left(Q-\frac{1}{A R} \tau_{23}\right)_{(l)} \\
+\left(1-\alpha^{2}\right) \tau_{23(l)}+\alpha^{2} \tau_{23(l-1)}
\end{gathered}
$$

Here, $\alpha=\Delta z_{l-1} / \Delta z_{l}[23]$. Assuming that $\tau_{23}$ and $\tau_{13}$ at $I=1$ are equal to zero for a shear free surface, the values of $\tau_{23}$ and $\tau_{13}$ at $I=2$ are calculated using eqn (17). Knowing the values now, at two points, the transverse shear stresses at all the other points in through-the-thickness directions are computed using eqn (18). At the interface, while setting up the equation, the values of $P$ and $Q$ are taken as the average of that at the top of the first layer and at the bottom of the second layer.

\section{NUMERICAL EXAMPLES}

A computer program is developed based on the theoretical models described earlier, for pointwise estimation of interlaminar transverse shear stresses in a composite cylindrical shell. To validate the accuracy of these models, some examples involving different material properties and fibre orientations are solved. The results obtained are compared with those available in the literature. The selective integration method has been adopted to calculate the contributions of membrane, bending and shear to the stiffiness of the element. 


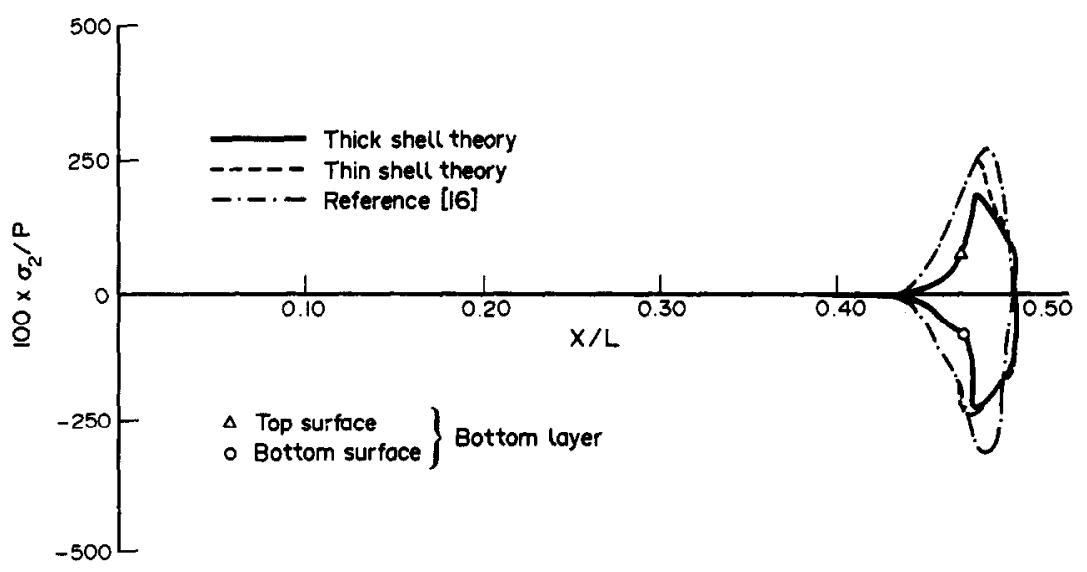

Fig. 7c. Variation of $\sigma_{2}$ along the length for $\left(0^{\circ} / 90^{\circ}\right)$ lamination.

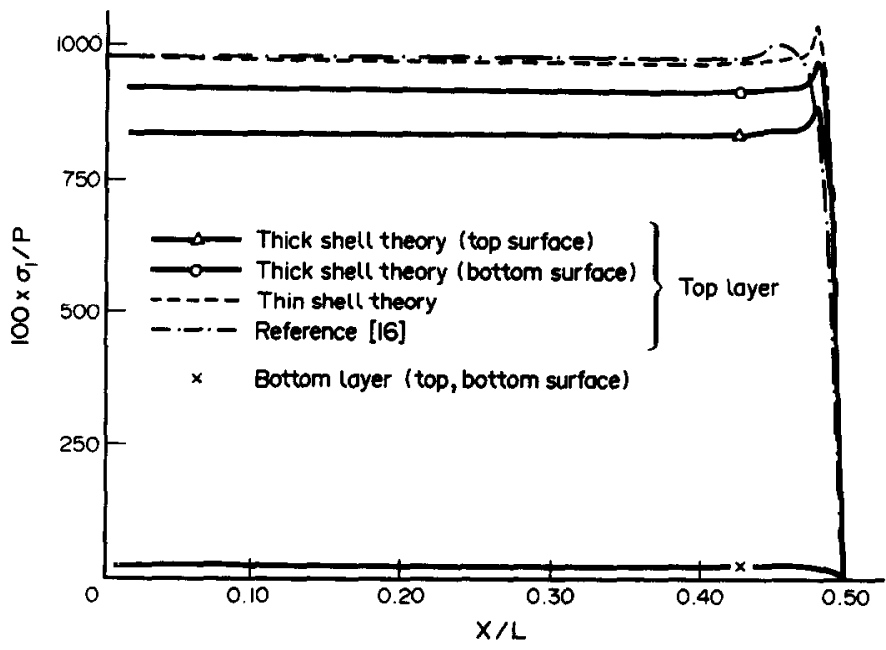

Fig. 7d. Variation of $\sigma_{1}$ along the length for $\left(0^{\circ} / 90^{\circ}\right)$ lamination.

\section{Example 1}

A two-layered angle-ply $\left(-15^{\circ} / 15^{\circ}\right)$, square plate, fixed at all four edges and subjected to a uniform distributed load of unity is considered. The material properties of the lamina are as follows.

$$
\begin{array}{r}
E_{1}=40 \times 10^{6} ; \quad E_{2}=10^{6} ; \quad G_{12}=G_{13}=0.5 \times 10^{6} ; \\
G_{23}=0.6 \times 10^{6} ; \quad v_{12}=v_{23}=v_{13}=0.25 .
\end{array}
$$

The plate is of unit length and the thickness is equal to 0.1 , as shown in Fig. 3a.

Because of asymmetrical behaviour, the full plate is discretized into 16 elements. The variations of displacement, normal stresses and transverse shear stress through the thickness are compared with higher-order plate theory and are plotted in Figs $4 a-4 d$.

\section{Example 2}

The problem of an infinite circular cylindrical shell subjected to periodically spaced band loads is investigated. Detailed comparison of the resulting stresses and displacements with exact elasticity
(E), Reissner-Naghdi (R-N) and Flugge shell (F) solutions [2] is carried out. The shell is made of an isotropic material with $E=10$ and $v=0.3$. The dimensions of the shell are shown in Fig. $3 b$.

The plots for the variation of displacements and the stresses across the thickness at different points along the length are presented in Figs 5a-5k.

The values of transverse shear stresses evaluated both by constitutive relation (CR) and the forward difference technique (FD) for geometrically thick shell theory and thin shell theory have been presented and compared with other solutions. Because of symmetry, only one-quarter of the shell is discretized for the analysis.

\section{Example 3}

A three-layered symmetrically laminated, crossply, simply supported cylindrical shell is investigated. The fibre orientations of the layers are $90^{\circ} / 0^{\circ} / 90^{\circ}$. The layers are of equal thickness. The geometry and the material properties of the shell are as follows (see Fig. 3 c): $L=200$ in; $R=10$ in; $h=2.0 \mathrm{in} ; E_{1}$ and $E_{2}$, the Young's moduli in the directions parallel 


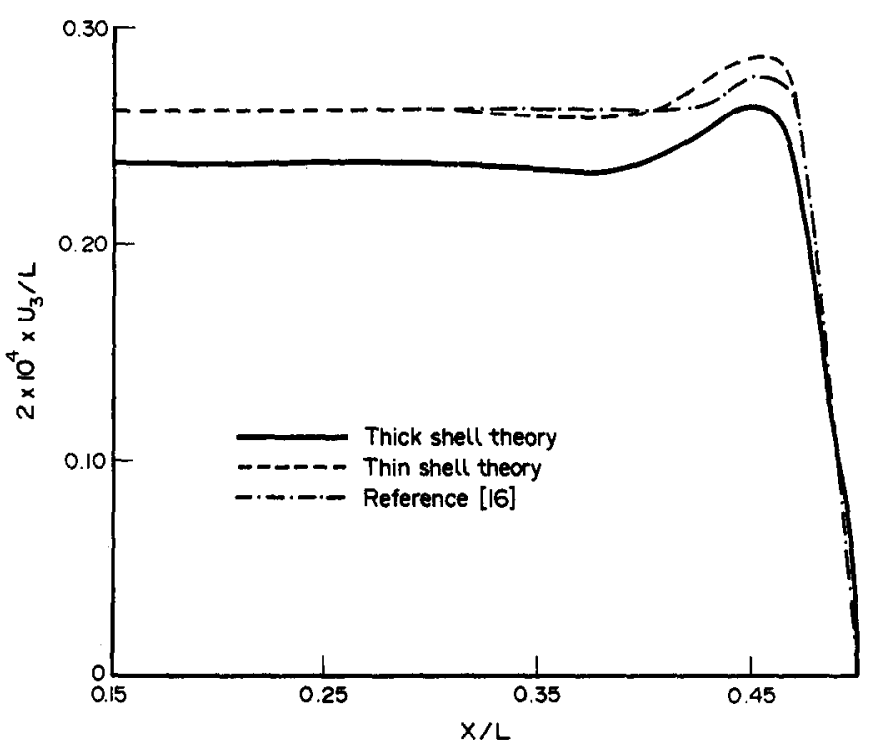

Fig. 8a. Variation of $U_{3}$ along the length for $\left(-45^{\circ} / 45^{\circ}\right)$ lamination.

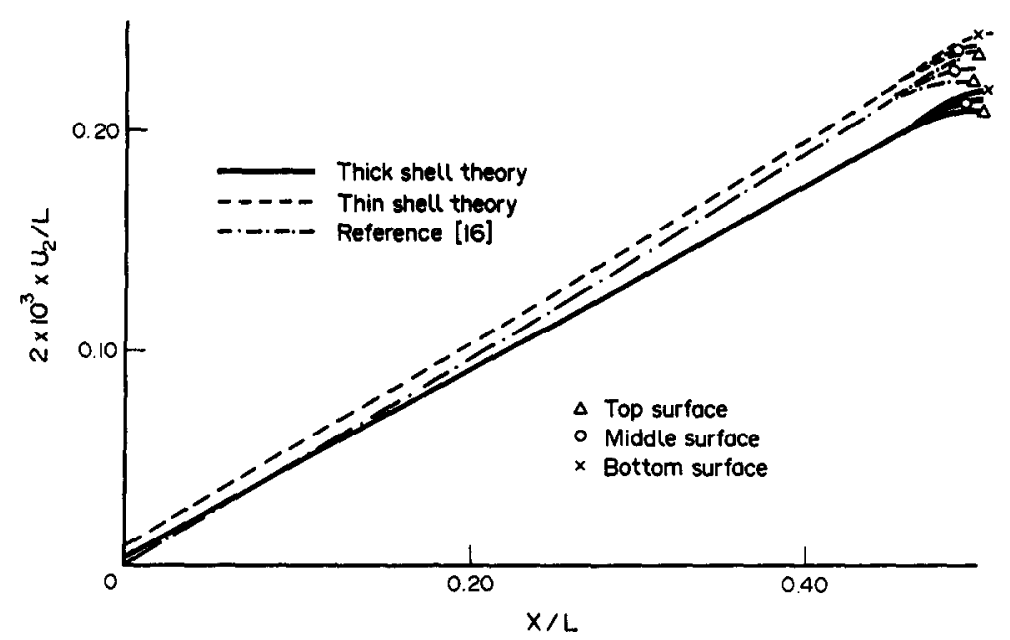

Fig. 8b. Variation of $U_{2}$ along the length for $\left(-45^{\circ} / 45^{\circ}\right)$ lamination.

and transverse to the fibres, are $40 \times 10^{6}$ and $10^{6}$ psi respectively; $G_{12}=G_{23}=G_{31}=0.5 \times 10^{6} \mathrm{psi}$; and $v_{12}=v_{23}=v_{13}=0.25$. The shell is subjected to an internal pressure of 100 psi. Because the shell is

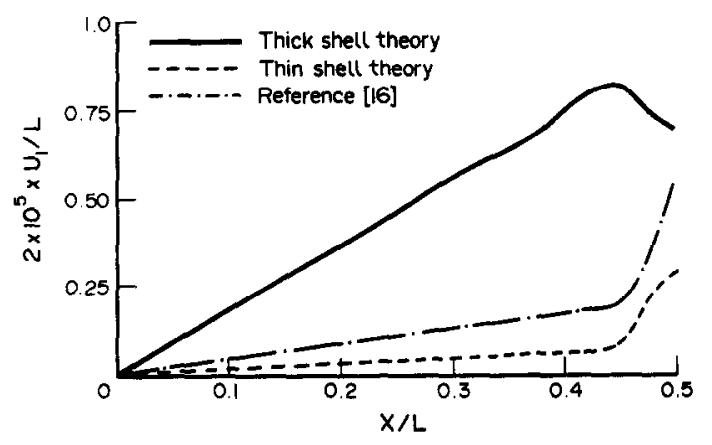

Fig. 8c. Variation of $U_{1}$ along the length for $\left(-45^{\circ} / 45^{\circ}\right)$ lamination. axisymmetrically loaded and symmetrically layered, only one-quarter of the shell is discretized with proper boundary conditions. The variation, of different parameters along the length of the shell are plotted and compared with exact solutions [16] in Figs 6a-6d.

\section{Example 4}

A two-layered asymmetric cross-ply cylindrical shell, simply supported at the ends, is considered. The fibre orientations of the outer/inner layer are $0^{\circ} / 90^{\circ}$. The layers are of equal thickness. The geometry and material properties of the layer are the same as in example 3. As the shell is axisymmetric with symmetric loading and the layers are cross-ply, one-quarter of the shell is discretized. The results obtained are plotted in Figs $7 \mathrm{a}-7 \mathrm{~d}$, and are compared with exact solutions given in [16]. 


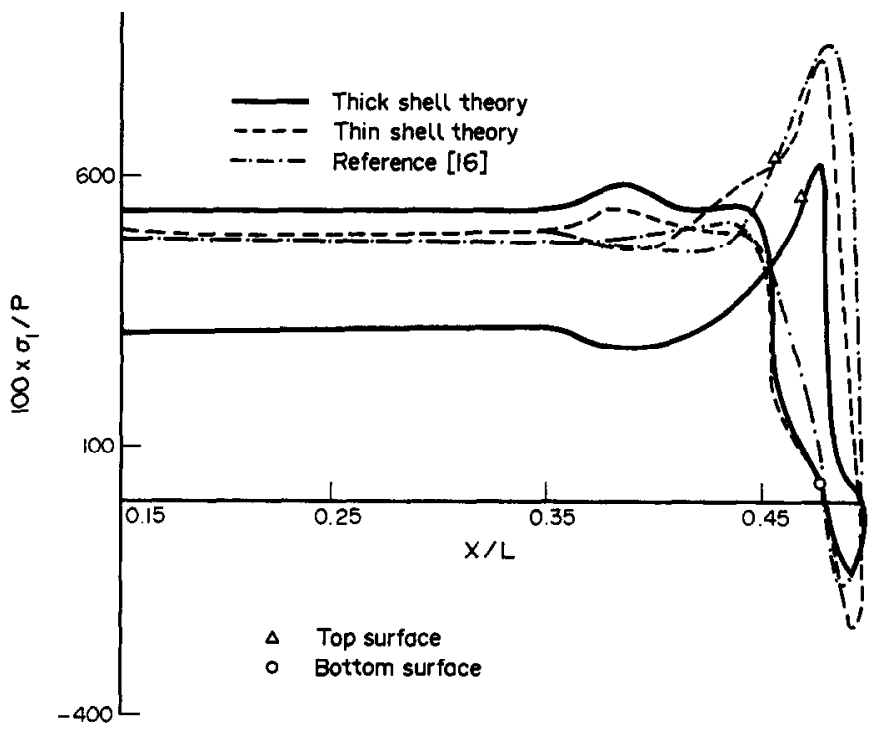

Fig. 8d. Variation of $\sigma_{1}$ along the length for $\left(-45^{\circ} / 45^{\circ}\right)$ lamination (top layer).

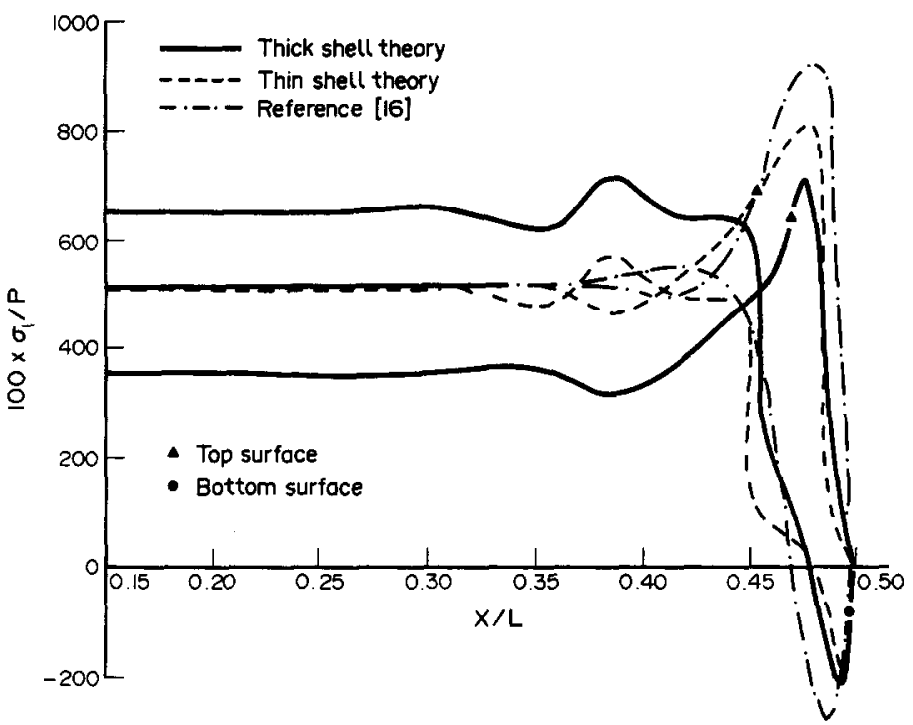

Fig. 8e. Variation of $\sigma_{1}$ along the length for $\left(-45^{\circ} / 45^{\circ}\right)$ lamination (bottom layer).

\section{Example 5}

A two-layered asymmetric angle-ply, circular cylindrical shell, simply supported, with fibre orientations of $45^{\circ} /-45^{\circ}$ for the outer and inner layers, is considered. The shell dimensions and the material properties are as in example 3. As the layers are asymmetric angle-ply, the full shell is discretized in the circumferential direction, and along the length, half the shell length is discretized, with the appropriate boundary condition for symmetry and supports at the ends. The results obtained are presented in Figs $8 \mathrm{a}-8 \mathrm{e}$, together with exact shell solutions [16] for comparison.

\section{CONCLUSIONS}

The results from a set of higher-order theories (geometrically thin shell and geometrically thick shell) for a composite cylindrical shell subjected to different loadings and boundary conditions are presented. These theories do not require the usual shear correction coefficients.

To validate these theories, a plate problem is first solved and the results are compared with a higherorder plate theory as shown in Figs $4 a-4 d$. It is seen that transverse displacement, variation of in-plane stresses and interlaminar stresses $\tau_{13}$ by constitutive relations, match across the thickness. The values of 
transverse shear stresses computed by a finite difference scheme, utilizing the equilibrium equations, are slightly lower than those given by the higherorder plate theory. This can be improved by taking closer pivot points and by increasing the number of subdivisions in each layer.

The influence of the ratio $h / R$ in a thick shell is pronounced, as seen in Figs $5 \mathrm{a}-5 \mathrm{k}$ of example 2 . The thick shell theory solutions are close to the elasticity theory solutions, as compared with the thin shell theory solutions, which are close to the results based on the Flugge shell theory. The transverse shear stress distributions from the thick shell theory are in excellent agreement with those of the elasticity theory and Reissner-Naghdi theory. The values of transverse shear stresses obtained by the elasticity theory have a peak near the inner shell surface, whereas those obtained by other theories have a peak near the centre of the shell thickness.

The cross-ply and angle-ply shells show a drastic redistribution of stresses in the shell as a result of the layering effect and anisotropy. Numerical results have been presented for $90 / 0 / 90,0 / 90$ and $-45 / 45$ laminations. The solutions obtained by the present theories have been compared with results from the available constant shear angle theory (CST), an extension of Mindlin's shear deformation theory for a homogeneous isotropic plate. It has been observed that the thin shell theory solutions are closer to CST solutions than are the thick shell solutions. For all laminations, transverse displacement $u_{3}$, longitudinal displacement $u_{2}$ and longitudinal stress $\sigma_{2}$ are the same for the three solutions in the central region, whereas the same is not true in the edge region. This is mainly because the central region has primarily a membrane action, whereas a bending action is predominant in the edge region. The slight variation of the transverse displacement obtained by the thick shell theory and the thin shell theory in the central regions is merely the result of the assumption that the loadings act on the mid-surface in the case of the thin shell theory and on the linear surface in the thick shell theory; this is actually the case. There is appreciable difference in the variations of circumferential stresses obtained by the thick shell theory and the thin shell theory. This implies that the influence of the ratio $h / R$ is predominant in the case of circumferential stresses and circumferential moments.

It may be concluded that the geometrically thick theory solutions are more reliable and accurate than those of the thin shell theory for both thin and moderately thick shells.

\section{REFERENCES}

1. H. Kraus, Thin Elastic Shells. John Wiley, New York (1967).

2. J. M. Klosner and H. S. Levine, Further comparison of elasticity and shell theory solutions. AIAA Jnl 4, $467-480$ (1966).
3. S. A. Ambartsumyan, Theory of Anisotropic Shells. Moscow (1961). English translation, NASA TT F-118 (1964).

4. P. M. Naghdi, A survey of recent progress in the theory of elastic shells. J. appl. Mech. Rev. 9, 365-368 (1956)

5. C. W. Bert and P. H. Francis, Composite material mechanics: structural mechanics. AlAA Jnl 12(9), 1173-1186 (1974).

6. R. A. Chaudhuri, Structural behaviour of FRP rectangular plate and cylindrical shells. M. Tech. Thesis, Department of Aeronautical Engineering, I.I.T., Madras (1974).

7. S. B. Dong and R. L. Taylor, On the theory of laminated anisotropic shells and plate. J. Aerospace Sci. 29, 969-975 (1962).

8. G. E. O. Widera and S. W. Chung, A theory of non-homogeneous anisotropic cylindrical shells. J. appl. Mech., ASME 21, 378-399 (1970).

9. R. C. Reuter, Analysis of shells under internal pressure J. Composite Mater. 6, 94-113 (1972).

10. K. Balaraman, V. X. Kunukkasseril and R. A. Chaudhari, Bending of asymmetrically laminated anisotropic shells subjected to internal pressure. Presented at 1st Conf. Reinforced Plastics and Their Aerospace Applications, Vikram Sarabhai Space Center, ISRO, Trivandrum (1972).

11. R. A. Chaudhuri, K. Balaraman and V. Kunukkasserial, Arbitrary laminated anisotropic cylindrical shells under internal pressure. AIAA Jnl 24(11), 1851-1857 (1986).

12. S. B. Dong and F. K. W. Tso, On a laminated orthotropic shell theory including transverse shear deformation. J. appl. Mech., ASME 39, 1091-1097 (1972).

13. R. D. Mindlin, Influence of rotatory inertia and shear on flexural motions of isotropic elastic plates. J. appl. Mech., ASME 18, 31 (1959).

14. J. N. Reddy, Exact solution of moderately thick laminated shells. J. Engng Mech., Div., ASCE 108, 794-809 (1984).

15. P. Seide and R. A. Chaudhuri, Triangular finite element for analysis of thick laminated shells. Int. J. Numer. Meth. Engng 24, 1563-1579 (1987).

16. Abu-Arja Kamal, Static analysis of laminated fiber reinforced plates and shells with shear deformation. $\mathrm{Ph} . \mathrm{D}$. Thesis, Department of Civil Engineering, University of Utah (1987).

17. J. M. Whitney and C. T. Sun, A refined theory for laminated anisotropic cylindrical shells. J. appl. Mech., ASME 41, 471-476 (1974).

18. T. Kant, Thick shells of revolution- - some studies. Ph.D. Thesis, Department of Civil Engineering, I.I.T., Bombay (1976).

19. A. Bhimaraddi, A higher order theory for free vibration analysis of circular cylindrical shells. Int. J. Solids Struct. 20(7), 623-630 (1984).

20. A. Bhimaraddi and L. K. Stevens, On the higher order theories in plates and shells. Int. J. Struct. 6(1), 35-50 (1986).

21. A. V. K. Murty and T. S. R. Reddy, A higher order theory for laminated composite cylindrical shells. J. Aeronaut. Soc. India, 161-171 (1986).

22. T. Kant and M. P. Menon, Higher-order theories for composite and sandwich cylindrical shell with $C^{0}$ finite elements. Comput. Struct. 33, 1191-1204 (1989).

23. M. G. Salvadori and M. L. Baron, Numerical Methods in Engineering. Prentice-Hall, Englewood Cliffs, NJ (1961).

24. B. N. Pandya, Higher order theories and finite element evaluation for multilayered composite plates. $\mathrm{Ph} . \mathrm{D}$. Thesis, Department of Civil Engineering, I.I.T., Bombay (1987).

25. G. E. O. Widera and D. Logan, Refined theories for nonhomogeneous anisotropic cylindrical shells, Part I. Derivation. J. Engng Mech. Div., ASCE 106, 1053-1074 (1980). 


\section{APPENDIX A}

The elements of the matrix for the geometrically thin shell theory are given here as follows. If we set

$$
H_{i}=\left(h_{L+1}^{i}-h_{L}^{i}\right) / i
$$

such that $i$ takes an integer value from one to seven, then the sub-matrices can be readily obtained in the following forms based on the geometrical assumption $(h / R) \ll 1$ :

$$
\begin{aligned}
& \mathrm{D}_{M}=\sum_{L=1}^{N L}\left[\begin{array}{llllll}
H_{1} Q_{11} & H_{1} Q_{12} & H_{1} Q_{13} & H_{3} Q_{11} & H_{3} Q_{12} & H_{3} Q_{13} \\
& H_{1} Q_{22} & H_{1} Q_{23} & H_{3} Q_{21} & H_{3} Q_{22} & H_{3} Q_{23} \\
& & H_{1} Q_{33} & H_{3} Q_{31} & H_{3} Q_{32} & H_{3} Q_{33} \\
& & & H_{5} Q_{11} & H_{5} Q_{12} & H_{5} Q_{13} \\
& & & & H_{5} Q_{22} & H_{5} Q_{23} \\
\text { Symmetric } & & & & H_{5} Q_{33}
\end{array}\right]^{\text {layer }} \\
& \mathbf{D}_{s}=\sum_{L=1}^{N L}\left[\begin{array}{cccccc}
H_{1} Q_{44} & H_{1} Q_{45} & H_{3} Q_{44} & H_{3} Q_{45} & H_{2} Q_{44} & H_{2} Q_{45} \\
& H_{1} Q_{55} & H_{3} Q_{54} & H_{3} Q_{55} & H_{2} Q_{54} & H_{2} Q_{55} \\
& & H_{5} Q_{44} & H_{5} Q_{45} & H_{4} Q_{44} & H_{4} Q_{45} \\
& & & H_{5} Q_{55} & H_{4} Q_{54} & H_{4} Q_{55} \\
& & & & H_{3} Q_{44} & H_{3} Q_{45} \\
\text { Symmetric } & & & & H_{3} Q_{55}
\end{array}\right]^{\text {Lth layer }}
\end{aligned}
$$

The elements of the $\mathrm{D}_{c}$ matrix are obtained by replacing $H_{1}, H_{3}$ and $H_{5}$ by $H_{2}, H_{4}$ and $H_{6}$ respectively in the $\mathrm{D}_{M}$ matrix above. Similarly, the $\mathbf{D}_{B}$ matrix is obtained by replacing $H_{1}, H_{3}$ and $H_{5}$ by $H_{3}, H_{5}$ and $H_{7}$ respectively in the $\mathbf{D}_{M}$ matrix.

\section{APPENDIX B}

The elements of the matrix for the thick shell theory are defined here as follows. If we set

$$
\begin{aligned}
H_{i} & =\left(h_{L+1}^{i}-h_{L}^{i}\right) / i \\
H_{i}^{-} & =\left(H_{i}-K \cdot H_{i+1}\right) \\
H_{i}^{+} & =\left(H_{i}+K \cdot H_{i+1}\right), \text { where } K=\frac{1}{R},
\end{aligned}
$$

and $i$ takes integer value from one to eight, then the sub-matrices can be readily obtained in the following forms based on the geometrical assumption $(h / R)^{2} \ll 1$ :

$$
\begin{aligned}
& \mathrm{D}_{M}=\sum_{L=1}^{N L}\left[\begin{array}{llllllll}
H_{1}^{-} Q_{11} & H_{1} Q_{12} & H_{1}^{-} Q_{13} & H_{1} Q_{13} & H_{3}^{-} Q_{11} & H_{3} Q_{12} & H_{3}^{-} Q_{13} & H_{3} Q_{13} \\
& H_{1}^{+} Q_{22} & H_{1} Q_{23} & H_{1}^{+} Q_{23} & H_{3} Q_{21} & H_{3}^{+} Q_{22} & H_{3} Q_{23} & H_{1}^{+} Q_{23} \\
& & H_{1}^{-} Q_{33} & H_{1} Q_{33} & H_{3}^{-} Q_{31} & H_{3} Q_{32} & H_{3}^{-} Q_{33} & H_{3} Q_{33} \\
& & & H_{1}^{+} Q_{33} & H_{3} Q_{31} & H_{3}^{+} Q_{32} & H_{3} Q_{33} & H_{3}^{+} Q_{33} \\
& & & & H_{5}^{-} Q_{11} & H_{5} Q_{12} & H_{5}^{-} Q_{13} & H_{5} Q_{13} \\
& & & & & H_{5}^{+} Q_{22} & H_{5} Q_{23} & H_{5}^{+} Q_{23} \\
\text { Symmetric } & & & & & H_{5}^{-} Q_{33} & H_{5} Q_{33} \\
& & & & & & & H_{1}^{+} Q_{33}
\end{array}\right] \\
& \mathbf{D}_{s}=\sum_{L=1}^{N L}\left[\begin{array}{lllllll}
H_{1}^{+} Q_{44} & H_{1} Q_{45} & H_{3}^{+} Q_{44} & H_{3} Q_{45} & H_{2}^{+} Q_{44} & H_{2} Q_{45} & H_{4} Q_{45} \\
& H_{1}^{-} Q_{55} & H_{3} Q_{54} & H_{3}^{-} Q_{55} & H_{2} Q_{54} & H_{2}^{-} Q_{55} & H_{4}^{-} Q_{55} \\
& & H_{5}^{+} Q_{44} & H_{5} Q_{45} & H_{4}^{+} Q_{44} & H_{4} Q_{45} & H_{6} Q_{45} \\
& & & H_{5}^{-} Q_{55} & H_{4} Q_{54} & H_{4}^{-} Q_{55} & H_{6}^{-} Q_{55} \\
& & & & H_{9}^{+} Q_{44} & H_{9} Q_{45} & H_{5} Q_{45} \\
& & & & & H_{9}^{-} Q_{55} & H_{5}^{-} Q_{55} \\
\text { Symmetric } & & & & & & H_{7}^{-} Q_{55}
\end{array}\right]
\end{aligned}
$$

The elements of the $\mathrm{D}_{c}$ matrix are obtained by replacing $\mathrm{H}_{1}, \mathrm{H}_{1}^{+}, \mathrm{H}_{1}^{-}, \mathrm{H}_{3}, \mathrm{H}_{3}^{+}, \mathrm{H}_{3}^{-}, \mathrm{H}_{5}, \mathrm{H}_{5}^{+}$and $\mathrm{H}_{5}^{-}$by $\mathrm{H}_{2}, \mathrm{H}_{2}^{+}, \mathrm{H}_{2}^{-}$, $H_{4}, H_{4}^{+}, H_{4}, H_{6}, H_{6}^{+}$and $H_{6}^{-}$respectively in the $\mathrm{D}_{M}$ matrix above. Similarly, the $\mathrm{D}_{B}$ matrix is obtained by replacing $H_{1}$, $H_{1}^{+}, H_{1}^{-}, H_{3}, H_{3}^{+}, H_{3}^{-}, H_{5}, H_{5}^{+}$and $H_{5}^{-}$by $H_{3}, H_{3}^{+}, H_{3}^{-}, H_{5}, H_{5}^{+}, H_{5}^{-}, H_{7}, H_{7}^{+}$and $H_{7}^{-}$respectively in the $\mathrm{D}_{M}$ matrix. 